CURRENT STATUS OF COMPUTATIONAL METHODS FOR TRANSONIC UNSTEADY AERODYNAMICS AND AEROELASTIC APPLICATIONS

John W. Edwards

John B. Malone

January 1992

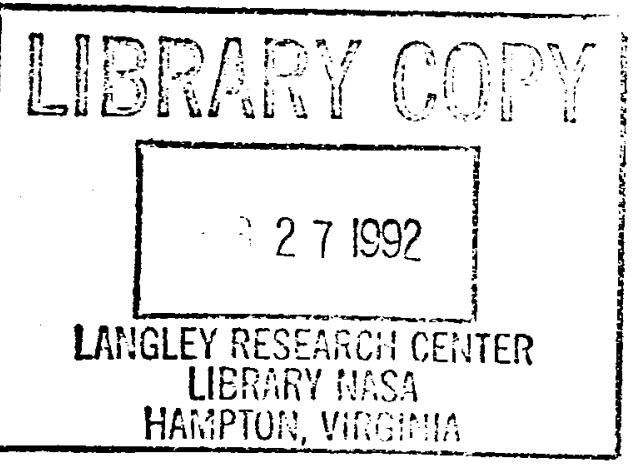




\title{
CURRENT STATUS OF COMPUTATIONAL METHODS FOR TRANSONIC UNSTEADY AERODYNAMICS AND AEROELASTIC APPLICATIONS
}

\author{
by \\ John W. Edwards \\ and \\ John B. Malone \\ NASA Langley Research Center \\ Hampton, Virginia 23665-5225 USA
}

\begin{abstract}
The current status of computational methods for unsteady aerodynamics and aeroelasticity is reviewed. The key features of challenging aeroelastic applications are discussed in terms of the flowfield state: low-angle high speed flows and high-angle vortex-dominated flows. The critical role played by viscous effects in determining aeroelastic stability for conditions of incipient flow separation is stressed. The need for a variety of flow modeling tools, from linear formulations to implementations of the Navier-Stokes equations, is emphasized. Estimates of computer run times for flutter calculations using several computational methods are given. Applications of these methods for unsteady aerodynamic and transonic flutter calculations for airfoils, wings and configurations are summarized. Finally, recommendations are made concerning future research directions.
\end{abstract}

\section{Introduction}

In the past decade there has been much activity in the development of computational methods for the calculation of unsteady aerodynamics about airfoils, wings and complete vehicle configurations. Two key areas of activity have been transonic aeroelasticity and lower speed, high-angle flight conditions. Advances have paralleled developments in steady Computational Fiuid Dynamics (CFD) with a lag of approximately five years due to the additional requirement of time-accuracy. This paper presents a discussion of current aeroelasticity problem areas or challenges. The focus is primarily upon methods aimed at the study of nonlinear fluid dynamic flows, typically referred to as Computational Fluid Dynamics (CFD), although attention is also given to linear flow models.

Figure 1 (Edwards [53]) illustrates significant features which must be addressed in the use of computational aeroelasticity for flutter boundary prediction. In this figure, a typical flutter boundary curve, characterized by the flutter speed gradually dropping to a minimum in the transonic speed range followed by a rapid upward rise, is shown. The ability to predict this minimum, termed the transonic flutter dip, is of great importance in design, since the flutter boundary must be shown by a combination of analysis and flight test to be outside the flight envelope by a specified margin. For military aircraft, the margin in terms of equivalent airspeed is at least 15 percent. Subsonic linear unsteady aerodynamic theories have been reasonably successful in predicting this flutter boundary for Mach numbers up to $0.6-0.7$ but linear theory is unable to account for the effects of aerodynamic shape and maneuvering condition upon unsteady airloads. At high Mach numbers linear analysis has been used with more or less success depending upon the severity of local transonic effects. The occurrence of flutter within the flight envelope of an aircraft usually leads to structural failure and loss of the vehicle, highlighting the necessity for careful validation of computational methods intended for use in this area. In addition, aircraft service life can be significantly degraded by unforeseen dynamic loadings, such as buffet, and predictive capability for such off-design point loadings must be well-understood before being utilized in structural design. These key differences in the utilization of steady and unsteady computational methods must be clearly understood.

This field received an initial impetus in the mid-1970's from three sources: Tijdeman's [152] experimental work on transonic unsteady pressure measurements, Magnus and Yoshihara's [108] demonstration of key transonic flow features for an airfoil with an oscillating flap and the introduction of an economical transonic finite-difference solution algorithm (LTRAN2) by Ballhaus and Goorjian [17]. Ballhaus [16] gives a survey of the field from this period. The AGARD Structures and Materials Panel Subcommittee on Aeroelasticity has selected experimental unsteady pressure data sets and defined two- and three-dimensional Standard Aeroelastic Configurations [30, 31] to provide reference computational test cases for the development and validation of improved computational methods.

Unsteady aerodynamics has been the theme of six recent conferences $[8-12,153]$ whose proceedings contain a wealth of information. Summary papers of the 1984 and 1985 AGARD conferences are given by Mykytow [114] and Mabey and Chambers [105]. The latter reference makes recommendations regarding computational and experimental methods for unsteady flow phenomena and draws particular attention to the need to pay careful attention to the nature of shock motions. The periodic oscillations about circular are airfoils are recommended as benchmark computational cases for all time-dependent transonic viscous flow theories. Zwaan [167] surveys aeroelastic problems in transonic flow while Deiwert [46] reviews the numerical simulation of unsteady interactive flows. Reference [155] provides a collection of articles going into extensive detail for unsteady transonic aerodynamics. Mabey [106] gives a review of pertinent experimental research on time-dependent aerodynamics. Finally, Dowell [48] provides an overview of nonlinear aeroelasticity phenomena including structural as well as aerodynamic nonlinearities. 


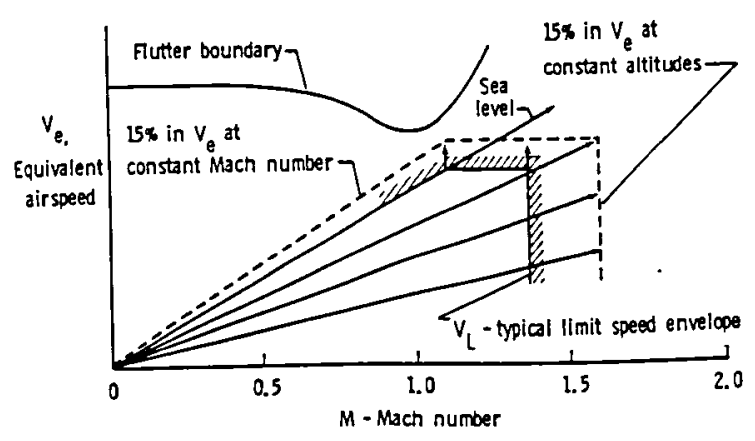

Figure 1 Graphical Representation of Minimum Required Flutter Margin for Military Aircraft [7].

Research in these areas requires the comparison of experimental and computational results with the goal of achieving accurate predictive capability. Edwards $[52,54]$ provides surveys of these efforts for the transonic flutter problem while Mabey[107] discusses the physical phenomena associated with unsteady transonic flow. Bobbitt's [36] review of the issues involved in obtaining accurate results from experiment and from computation is particularly noted. Regarding higher angle, vortex dominated flows, a trend of increasing interest by the aerodynamics community in unsteady flows is also noted. This is due to the inherent unsteadiness of such flows and to the ability of emerging CFD methods to simulate their details. Newsome and Kandil [117] discuss physical modeling issues involved in the computational prediction of vortex dominated flows and survey numerical results.

The remainder of this paper will review the current status of computational methods for unsteady aerodynamics and aeroelasticity. The key features of challenging aeroelastic applications are discussed in terms of the flowfield state: low-angle high speed flows and high-angle vortex-dominated flows. Next the computational methods and the basic fluid dynamic equations are introduced, followed by an assessment of the computer re-

sources required for the unsteady aerodynamic computations. Then, the current state-of-the-art in CFD methods for transonic flows and vortex-dominated flows are each discussed, with emphasis in the progress achieved during the past half decade. Finally, an assessment of current capabilities and future research trends is offered.

\section{Features of Low-Angle, High Speed Flows}

The main features of steady transonic flow are described first in order to organize the discussion. With increasing Mach number and moderate angle-of-attack, the flow over the upper surface of an airfoil becomes critical between $M_{\infty}=0.4-0.7$ with the first shocks forming at approximately 0.1 higher Mach number. Pearcy et al. [120] have classified several types of flow separation which may occur. For conventional airfoils the typical pattern involves the growth of a local separation bubble, induced by boundary layer separation at the shock foot, spreading rapidly to the trailing edge as Mach numbers increases. This condition is often accompanied by unsteady phenomena such as buffet and aileron buzz (Tijdeman [152]).

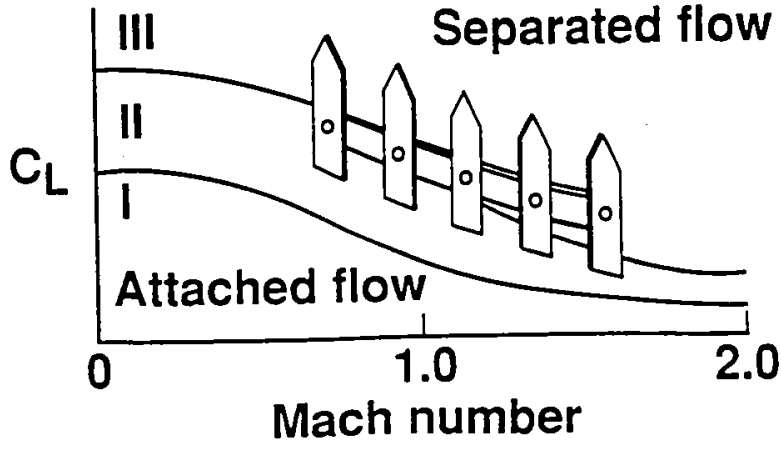

Figure 2 Characteristics of Attached and Separated Flow for Complete Aircraft (Edwards [53]).

The steep aft pressure gradients of modern airfoils can lead to an alternate pattern in which separation progresses from the trailing edge towards the shock. Tijdeman [152] notes that the flow conditions in the region between the onset of trailing edge separation and fully separated flow are very sensitive to Reynolds number and the location of transition from laminar to turbulent flow.

Figure 2 shows a diagram, from Edwards [53], of attached, mixed and separated flow regions for a complete aircraft at freestream Mach numbers between 0 and 2.0. In region 1 , the flow is predominantly attached. To obtain optimum performance and to avoid the drag penalty associated with flow separation, design cruise conditions for aircraft typically are located here near the boundary of region II.

As speed and/or angle of attack increase, a transition region of mixed flow (region II of fig. 2) is encountered. For rigid structures, this region is typified by the onset of localized regions of flow separation which may exhibit significant aerodynamic unsteadiness. For realistic flexible structures, the aeroelastic response of the structure interacts with the airflow to induce much more complicated situations. For instance, structural vibrations can cause the flow to alternately separate and reattach at flow conditions where a rigid structure would support attached flow. The associated high unsteady aerodynamic loading can interact with the structure to cause unusual aeroelastic phenomena which may restrict the vehicle flight envelope.

With further speed and/or angle of attack increases which may be encountered under maneuvering conditions, fully separated flow conditions emerge (region III of fig. 2). Leadingedge vortex flows and stalled wing flows are of this nature. At still higher angles, vortex bursting in the vicinity of the aircraft can cause severe buffeting. Within such regions the flow is highly unsteady and accurate computations will require careful attention to turbulence modeling. To emphasize the complexity which the aeroelastic response adds, the flow within the three regions of figure 2 will be referred to as Type I, II, and III respectively.

While the predictive methods for attached flows are reasonably well developed, the picket fence in figure 2 emphasizes the difficulty in predicting aeroelastic phenomena in the mixed and separated flow regions. It also symbolizes novel features that 
are being encountered in transonic flutter testing. Modem high performance aircraft are capable of maneuvering at transonic speeds, leading to a much enlarged parameter space that must be considered in flutter analysis and testing. Wing/store loading, fuselage interference, angle-of-attack, Reynolds number, wing shape, and wing sweep all must be considered, and the traditional flutter boundary parameterization of dynamic pressure at flutter versus Mach number may need to be augmented to adequately describe aeroelastic stability boundaries. For instance, flutter tests give some indication that these additional parameters affect the detailed aeroelastic stability condition near the flutter boundary. Thus, the pickets of the fence in figure 2 represent possible regions of low damping or instability that might be encountered.

\section{Features of High-Angle, Vortex-Dominated Flows}

Unsteady airloads due to flow separation are involved in a number of cases critical to the structural integrity of aircraft. As speed increases for moderate angles of attack, typical of maneuvering flight near trimmed flight conditions, local transonic flow effects are encountered which lead to separated flow over the aft portions of lifung surfaces. Minimum flutter speed indices are often encountered in this transonic region, in conjunction with the onset of separated flow. The ability to predict these minimums is obviously tied to the ability to treat such "local" separated flows on wings.

For slightly lower speeds where more aggressive maneuvering is possible, unsteady airloads due to flow separation over "remote" components (e.g. forebody and main wing panel) leads to issues of tail buffeting and structural fatigue of aircraft components. For these cases, as speed and/or angle of attack increase, smooth air flow over lifting surfaces breaks down in a variety of ways depending strongly upon the geometry. For lower sweep angles and blunt leading edges, flow separation may initiate near the trailing edge or near shocks and progress to completely separated and stalled conditions. For higher sweep angles and less blunt leading edges, leading edge flow separation bubbles foreshadow the development of leading edge vortex flows. At higher angles, unsteady and burst vortex flow in the vicinity of the wing and downstream lifting surfaces leads to strong unsteady airloads and buffeting. Flow conditions near the boundaries of these regions for the various flow phenomena can be sensitive to a number of conditions and an understanding of these effects is called for in order to avoid adverse aeroelastic effects such as stall flutter, buzz, and structural buffeting.

\section{Computational Aeroelasticity Challenges}

With figure 2 providing a framework within which typical flowfields encountered in aeroelasticity may be viewed, a number of current aeroelasticity problem areas are introduced in Table I and figures 3-7. Table I serves as a guide for discussing the current status in this area and the likely future trends. On the left are listed the key Challenges, most of which have been extensively commented on above. They are roughly graded in terms of increasing difficulty from top to bottom with the more difficult areas calling for more sophisticated flowfield modeling in order to achieve useful accuracies. Arrayed against these challenges are the Resource Issues impacting the economics of aeroelastic analysis, which are listed on the right. The choice of the appropriate level of CFD code to use, indeed the decision of whether to use a linear or nonlinear flow method, is dictated by

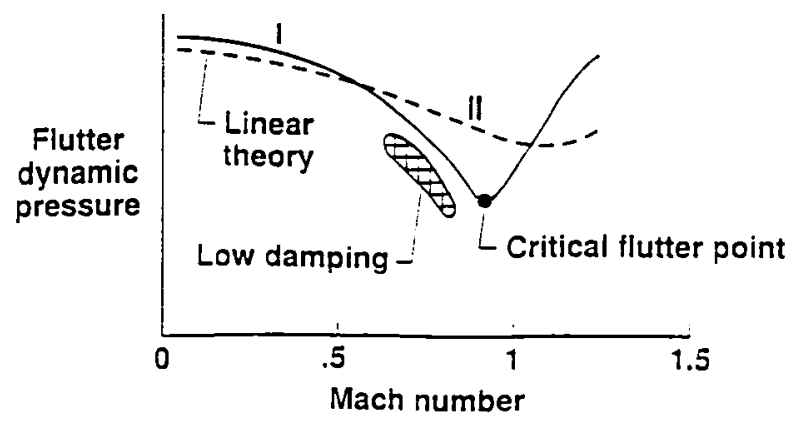

Figure 3 Features of Transonic Flutter (Edwards [53]).

the stage of design maturity, the required level of accuracy and computer resources available. The process of identifying critical loading conditions requires nunning large numbers of cases, almost always utilizing lower level methods. Critical cases, so identified, are then candidates for further analysis with higher level methods. It is interesting to query if this process can be relied upon to capture the actual critical loading cases.

Figure 3, from Edwands [53], indicates further features of high speed, low angle flutter. Dynamic pressure at flutter tends to decrease with increasing Mach number to a minimum "critical flutter point" value in the transonic speed range. At subsonic speeds where the flow can be assumed to be attached (Type I flow) at flutter, linear theory is reasonably accurate. As speed increases into the transonic region, the situation is complicated by the formation of shock waves and the onset of flow separation (Type II flow) and linear theory must be used with caution. The low damping region in the figure indicates the potential for nonclassical aeroelastic response and instabilities which may be encountered. Figures $4-6$, illustrate several types of novel aeroelastic responses which have been encountered with the onset of Type II flows and which offer challenges for computational methods. Figure 4 (Edwards [53]) shows a region of nonclassical aeroelastic response observed on a high aspect ratio, flexible, supercritical wing (Seidel et al. [141]) where high dynamic response at nearly constant Mach number was encountered at dynamic pressures well below those for which flutter was predicted with linear theory. The motion is of the limit-amplitude type and the response is believed to be associated with flow separation and reattachment driving the wing motion in the first bending mode. Figure 5 (Edwards [53]) illustrates wing/store limited amplitude oscillations experienced by modern, high performance aircraft under various loading and maneuvering conditions at transonic Mach numbers. Such oscillations can result in limitations on vehicle performance. The conditions for which this type of response occurs appear to also be near the onset of Type II mixed flow. The response typically increases for maneuvering flight conditions. Dynamic vortex-structure interactions causing wing oscillations have been observed, figure 6 (Dobbs et al. [47]), on a bomber type aircraft for high wing sweep conditions during wind-up turn maneuvers. The flow involves the interaction of the wing vorex system with the wing first bending mode and occurs over a wide Mach number range at moderate angles of attack. 
For lower speed flight where higher angles are achieved, fully separated flows (Type III flows in fig. 2) are encountered which can range from diffuse vortical flow structures to concentrated vortices designed to enhance stability and control. Interaction of such forebody and wing vonex systems with aft vehicle components results in vortex-induced buffet loads. Figure 7 (Edwards [53]) shows typical operating conditions at which such empennage buffet may be encountered. Buffet of horizontal tails can occur at intermediate angles of attack and is a result of the vortex system propagating downstream and encountering the horizontal tail surface. As angle of attack increases, the location of vortex bursting moves upstream in the wake. Loss of lift is associated with the burst location reaching the vicinity of the aircraft, and verical surfaces located in such regions can experience severe dynamic loads and structural fatigue.

These challenges, illustrated by figures $3-7$, involve two types of unsteady flows. The first is the Type II flow (fig. 2) wherein the onset of flow separation at high speeds leads to critical flutter conditions and/or novel aeroelastic responses. The second involves fully separated Type III vortex-dominated flows at high angles. The search for the appropriate levels of sophistication in fluid dynamic modeling to adequately model these flows is the subject of this paper.

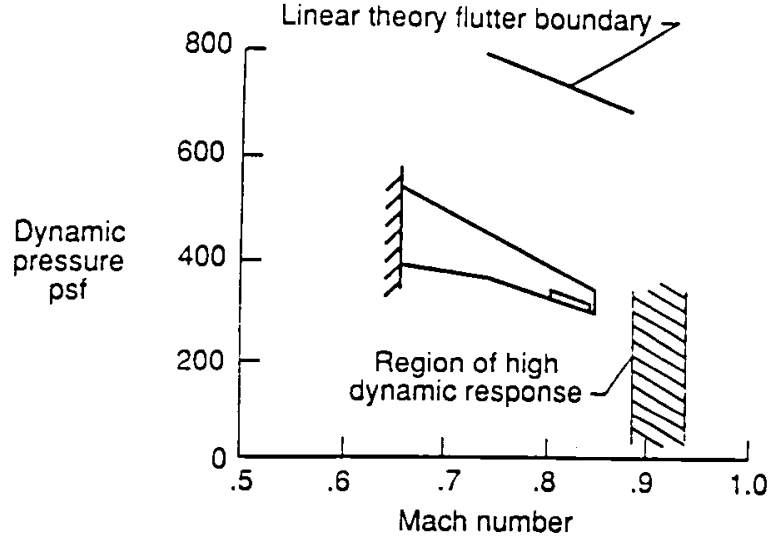

Figure 4 Region of High Dynamic Response Encountered During Test of a Flexible Supercritical Wing (Edwards [53]).

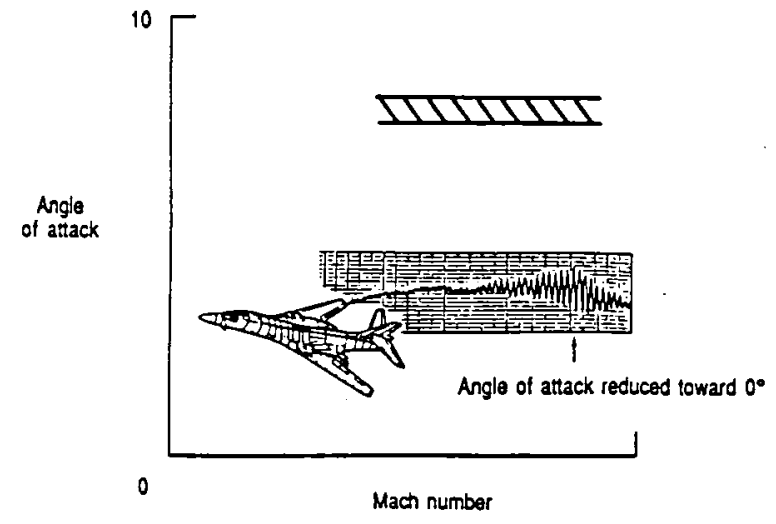

Figure 6 Vortex-Induced Wing Oscillations of a Bomber-Type Aircraft (Dobbs, Miller, and Stevenson [47]).
Table 1 COMPUTATIONAL AEROELASTICITY
CHALLENGES AND RESOURCE ISSUES

\begin{tabular}{|c|c|}
\hline Challenges & Resource Issues \\
\hline $\begin{array}{l}\text { Stability \& Control } \\
\text { divergence } \\
\text { roll performance } \\
\text { wing rock }\end{array}$ & $\begin{array}{l}\text { Modeling Tradeoffs } \\
\text { higher level CFD } \\
\text { improved configuration } \\
\text { detail }\end{array}$ \\
\hline $\begin{array}{l}\text { Gust Response } \\
\text { Flutter Boundary Prediction } \\
1-\mathrm{g} \\
\text { maneuvering } \\
\text { limit cycle oscillations }\end{array}$ & $\begin{array}{l}\text { Design Maturity } \\
\text { preliminary design } \\
\text { final design } \\
\text { critical loading conditions }\end{array}$ \\
\hline $\begin{array}{l}\text { Control Effectiveness } \\
\text { buzz } \\
\text { hinge moment }\end{array}$ & $\begin{array}{l}\text { Computer Resources } \\
\text { required level of accuracy } \\
\text { cost per solution } \\
\text { number of solutions }\end{array}$ \\
\hline $\begin{array}{l}\text { Buffet Response } \\
\text { local: main wing panel } \\
\text { remote: tail buffet }\end{array}$ & , \\
\hline
\end{tabular}

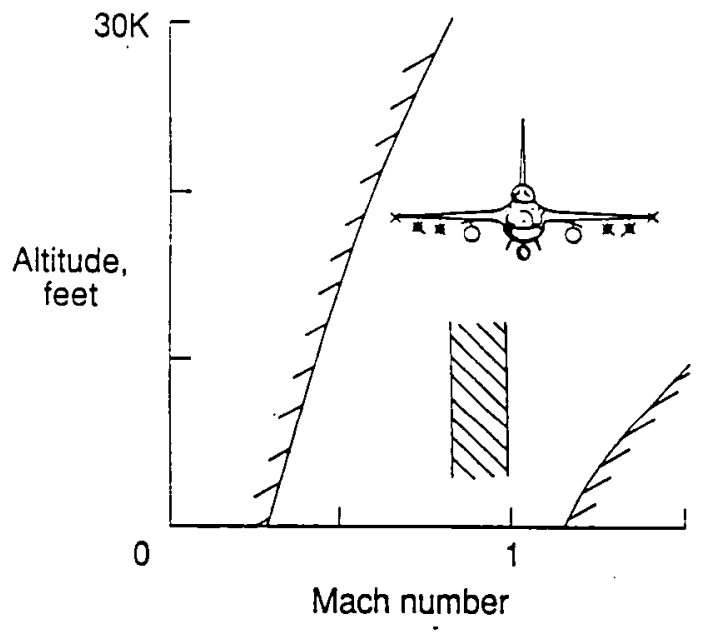

Figure 5 Region of Potential Limited-Amplitude Oscillations Due to Wing-Store Loading Effects (Edwards [53]).

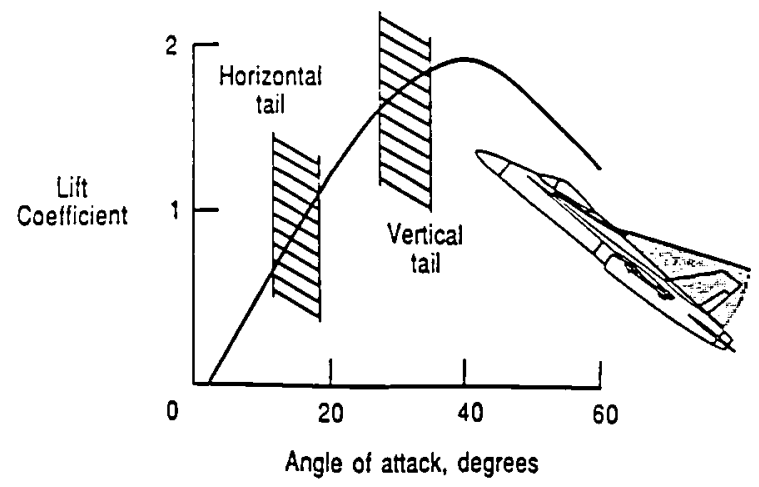

Figure 7 Regions of Vortex-Induced Buffet Loads (Edwards [53]). 


\section{Computational Methods}

A variety of fluid dynamic flow models is available to address unsteady aerodynamic computations. The choice of an appropriate method calls for assessment of the difficulty of the aerodynamic problem being addressed. Type I flows (fig. 1) include one of the most important aeroelastic analysis conditions, that of cruise at high dynamic pressure. Classical linear aeroelastic analysis has been primarily focused upon this condition. The transition from Type I to Type II conditions can occur due to aircraft maneuvering, with little decrease in dynamic pressure. Thus, aeroelastic response and stability of aircraft operating in Type II flows can be quite important although they have only begun to be brought within the range of computational methods.

Fluid dynamic flow models available for unsteady aerodynamic computation include: the classical (linear) small disturbance potential equation, the nonlinear potential equation (both Transonic Small Disturbance (TSD) and Full Potential equation (FPE)), the Euler equations (EE) and the Navier-Stokes equations (both Full (FNS) and thin-layer (TLNS)).

Issues which have been central to unsteady CFD have been the choice of implicit versus explicit algorithms, the stability of altemative solution algorithms and the treatment of computational grids. Explicit schemes are simple to code and easily vectorizable but are limited in allowable time step by the stability limit imposed by the signal propagation time over the smallest grid cell. Faced with the requirement of maintaining time-accuracy throughout the entire field for aeroelastic computations, this easily leads to excessive computation times, especially for viscous flow calculations where a very fine mesh near the surface is required to resolve the boundary layer. The alternative implicit solution algorithms thus are currently favored for present-day computer architectures because of their relative stability and time-step characteristics. While no attempt will be made to present complete details of the various levels of flow models, the following sections highlight the key equations and relevant boundary conditions.

\subsection{Navier-Stokes Equations}

Anderson, Tannehill and Pletcher [2] provide a description of the three-dimensional Full Navier-Stokes equations. For engineering applications, the Reynolds-averaged form of the FNS equations are normally used as a basis for practical computational procedures. In a cartesian coordinate system, the FNS equations can be written as follows:

$$
\frac{\partial}{\partial t}(Q)+\frac{\partial}{\partial x}\left(F-F_{v}\right)+\frac{\partial}{\partial y}\left(G-G_{v}\right)+\frac{\partial}{\partial z}\left(I I-H_{v}\right)=0
$$

where the vector of independent, conserved variables is

$$
Q=(\rho, \rho u, \rho v, \rho w, e)^{T}
$$

and

$$
\begin{gathered}
F=\left(\rho u, \rho u^{2}+p, \rho u v, \rho u w, u(e+p)\right)^{T} \\
G=\left(\rho v, \rho u v, \rho v^{2}+p, \rho v w, v(e+p)\right)^{T} \\
H=\left(\rho w, \rho u w, \rho v w, \rho w^{2}+p, w(e+p)\right)^{T}
\end{gathered}
$$

are the inviscid flux vectors. The viscous flux vectors, $F_{v}, G_{v}$ and $H_{v}$ are given in Ref. [2].

To facilitate solutions of the FNS equations on body-fitted computational grids, a curvilinear coordinate system is defined and eqs. (1) are converted using a generalized coordinate transformation of the type:

$\xi=\xi(x, y, z, t), \quad \eta=\eta(x, y, z, t), \quad \varsigma=\varsigma(x, y, z, t), \quad \tau=t$

into the following expressions:

$\frac{\partial}{\partial t}(\dot{Q})+\frac{\partial}{\partial \xi}\left(\hat{F}-\hat{F}_{v}\right)+\frac{\partial}{\partial \eta}\left(\hat{G}-\hat{G}_{v}\right)+\frac{\partial}{\partial \zeta}\left(\hat{H}-\hat{H}_{v}\right)=0$

where:

$$
Q=\frac{1}{J}(\rho, \rho u, \rho v, \rho w, e)^{T}
$$

$F=\frac{1}{J}\left(\rho U, \rho U u+\xi_{x} p, \rho U v+\xi_{y} p, \rho U w+\xi_{z} p, U(e+p)-\xi_{t} p\right)^{T}$

$\dot{G}=\frac{1}{J}\left(\rho V, \rho V u+\eta_{x} p, \rho V v+\eta_{y} p, \rho V w+\eta_{z} p, V(e+p)-\eta_{t} p\right)^{T}$

$H=\frac{1}{J}\left(\rho W, \rho W u+\zeta_{x} p, \rho W v+\zeta_{y} p, \rho W w+\zeta_{z} p, W(e+p)-\zeta_{t} p\right)^{T}$

Similar transformations are applied to the viscous flux vectors. Note that the transformation is time-dependent, allowing the grid to move to follow body motion and giving rise to grid motion terms such as $\xi_{t}, \eta_{t}$ and $\zeta_{t}$ in eqs. (9) to (11). For viscousflow aerodynamic computations, the solid-surface boundaries are modeled using the "no-slip" condition, together with adiabatic wall and zero normal pressure gradient conditions.

A modified form of the FNS equations, termed the thinlayer Navier-Stokes equations, has been found useful for applications where viscous effects in certain spatial directions are small enough to be neglected. For many aerodynamic flows of interest, the viscous terms normal to the body are of most importance, and the other viscous fluxes can be dropped. If the $\eta$-direction in eq. (7) is taken as the body normal direction, a TLNS form of eq. (7) is given by the expression:

$$
\frac{\partial}{\partial t}(\hat{Q})+\frac{\partial}{\partial \xi}(\hat{F})+\frac{\partial}{\partial \eta}\left(\hat{G}-\hat{G}_{v}\right)+\frac{\partial}{\partial \zeta}(\hat{H})=0
$$

For turbulent-flow calculations, turbulence modeling such as the algebraic eddy viscosity model of Baldwin and Lomax [15] is used. Rumsey and Anderson [134] are typical of applications using this thin-layer approximation to compute viscous-flow solutions for airfoils. Also, Thomas et al. [150] describe a three-dimensional implementation of the above equations in the CFL3D code.

\subsection{Euler Equations}

For aerodynamic flows in which viscous effects are expected to be negligible, the inviscid Euler equations can be derived from eq. (7) by dropping all three of the viscous flux vectors from the formulation. Then, $F_{v}=G_{v}=H_{v}=0$, and eq. (7) reduces to:

$$
\frac{\partial}{\partial t}(\hat{Q})+\frac{\partial}{\partial \xi}(\hat{F})+\frac{\partial}{\partial \eta}(\hat{G})+\frac{\partial}{\partial \zeta}(\hat{H})=0
$$


The boundary conditions appropriate to the Euler equations are the "slip" or "flow tangency" conditions. For these applications, only the velocity component normal to the body surface is set to zero. The flow streamlines are assumed to run parallel to the surface tangent at each point on the surface. Note that for situations where there is rotational flow, such as the regions behind strong or curved shock eaves, the Euler equations can propagate the vorticity downstream in a correct manner if an adequate number of grid points are used.

\subsection{Full Potential Equation}

The FPE is derived from the Euler equations by assuming that the flow is inviscid, isentropic and irrotational. A velocity potential can then be defined whose derivatives in the spatial directions recover the flow velocity components in the appropriate directions. The goveming equations for this formulation are the continuity equation:

$$
\rho_{t}+\left(\rho \Phi_{x}\right)_{x}+\left(\rho \Phi_{y}\right)_{y}+\left(\rho \Phi_{z}\right)_{z}=0
$$

and the isentropic energy equation:

$$
\rho=\left[1+\frac{\gamma-1}{2} M_{\infty}^{2}\left(1-2 \Phi_{t}-\Phi_{x}^{2}-\Phi_{y}^{2}-\Phi_{z}^{2}\right)\right]^{\frac{1}{\gamma-1}}
$$

As with the Euler equations, a generalized coordinate system is often used to solve the FPE and a flow-tangency boundary condition is enforced at the body surface. Sankar and Malone [136] describe such a FPE formulation in generalized coordinates.

\subsection{Transonic Small Disturbance Potential Equation}

The Transonic Small Disturbance Potential equation is derived from the inviscid Euler equations assuming that the flow is isentropic, irtotational and a small perturbation of a steady uniform flow, $U$, in the $\mathrm{x}$ direction. The TSD velocity potential function, $\phi$, describes the perturbed velocity components $u, v$, w:

$$
u=\frac{\partial \phi}{\partial x}, \quad v=\frac{\partial \phi}{\partial y}, \quad w=\frac{\partial \phi}{\partial z}
$$

where the total velocity in the $x$ direction is U+u. Batina [20] and Batina et al. [21] give the modified TSD potential equation in conservation form as

$$
\frac{\partial f_{0}}{\partial t}+\frac{\partial f_{1}}{\partial x}+\frac{\partial f_{2}}{\partial y}+\frac{\partial f_{3}}{\partial z}=0
$$

where

$$
\begin{array}{cll}
f_{0}=-A \phi_{t}-B \phi_{x} & ; & f_{2}=\phi_{y}+H \phi_{x} \phi_{y} \\
f_{1}=E \phi_{x}+F \delta_{x}^{2}+G \phi_{y}^{2} & ; & f_{3}=\phi_{z}
\end{array}
$$

The coefficients A-H are given by Batina [20]. The TSD equation (17) is distinguished from the higher equation level flow models in that, within the small disturbance assumption, the computational grid is not required to move with the body since boundary conditions are imposed at the mean plane, usually $z$ $=0^{ \pm}$. The wing flow tangency boundary condition is

$$
\phi_{z}^{ \pm}=f_{x}^{ \pm}+f_{t}
$$

where $f^{ \pm}(\mathrm{x}, \mathrm{y}, \mathrm{t})=0$ describes the upper and lower body surfaces. The trailing wake boundary conditions are

$$
\left[\phi_{x}+\phi_{t}\right]=0 \quad ; \quad\left[\phi_{z}\right]=0
$$

where $[-]$ indicates that jump in the indicated quantity across the wake. The pressure coefficient may be computed using either linear or nonlinear forms of the Bernoulli equation. Batina et al. [21] describe this algorithm as implemented in the CAP-TSD code with a number of example calculations.

\subsection{Viscous-Inviscid Interaction}

Neither the potential equations nor the Euler equations described above incorporate viscous effects which can be important for high speeds and for lower speed at higher angles. It is possible to account for unsteady viscous effects by coupling a viscous boundary-layer model with an otherwise inviscid analysis. As commonly implemented, the inviscid outer flow solution provides the surface pressure distribution needed to solve the boundary layer equations. This yields the boundary-layer displacement thickness distribution which is used to modify the airfoil surface tangency boundary condition for the next iteration of the outer inviscid flow solution.

Guruswamy and Goorjian [65], Howlett and Bland [81], and Rizzetta [129] describe this method implemented in twodimensional unsteady TSD codes. The effect of a viscous boundary layer for attached turbulent flow is modeled in a quasisteady manner by means of the lag-entrainment equations of Green et al. [64]. In this integral method the displacement thickness $\delta^{*}$ is computed as a function of the boundary-layer momentum thickness $\theta$ and the shape factor $\mathrm{H}$ as

$$
\delta^{*}=\theta \cdot H
$$

Given the velocity at the edge of the boundary layer (from the outer inviscid flow solution), the boundary layer equations may be integrated in a "direct" fashion to obtain $\delta^{*}$. Coupling between the boundary-layer and the outer inviscid flow is through the boundary conditions on the airfoil and wake, eqs. (19) and (20), which are modified to

$$
\phi_{z}^{ \pm}=f_{x}^{ \pm}+f_{t}+\left(\delta^{*} / c\right)_{x}^{ \pm} \quad ; \quad\left[\phi_{z}\right]=\left[\left(\delta^{*} / c\right)_{x}\right]
$$

For cases of incipiently separating and separated flows the boundary layer equations become singular, requiring a reformulation of the equations in an "inverse" mode in which the edge velocity gradient is computed for a given displacement thickness (East et al. [49]). Consistency with the outer inviscid flow may be obtained via a "semi-inverse" relaxation coupling method (in which $\delta^{*}$ is updated based upon the error between inner and outer edge velocities) described by Carter [38] and also used by Fenno et al. [58]. For cases with large amounts of separated flow, particularly for unsteady flow, the semiinverse method itself encounters stability problems (Edwards and Carter [50]). These cases have been more tractable via the "quasi-simultaneous" coupling method introduced by Veldman [158] and by Houwink and Veldman [79] and the "semiimplicit" coupling method of LeBalleur and Girodroux-Lavigne [98]. Both of these methods perform the viscous-inviscid coupling by developing, at each grid point, locally linear relations between the inner and outer flow variables. This enables simultaneous solution for the coupling variables which is usually accompanied by relaxation and iterations for convergence. The quasi-simultaneous method has been implemented using the low frequency LTRAN2-NLR TSD code and quasi-steady integral boundary layer equations. The semi-implicit method described in Ref. [98] achieves full time-consistency by coupling a timeaccurate TSD code with a time-accurate integral boundary layer method. 


\subsection{Time-Linearized Transonic Small Disturbance Equation}

A second formulation of the Transonic Small Disturbance Potential equation is the time-linearized equation, which is derived by assuming that the unsteady flowfield can be treated as a small perturbation about the steady flow field solution. This the ory assumes that shockwaves are neither created nor destroyed during the unsteady motion. The steady flow potential is obtained from solutions to the steady-flow version of the TSD equation:

$$
\left[1-M_{\infty}^{2}-(\gamma+1) M_{\infty}^{2} \phi_{x}^{o}\right] \phi_{x x}^{o}+\phi_{y y}^{o}+\phi_{z z}^{o}=0
$$

The unsteady potential, $\phi^{1}$, is then computed from the unsteady TSD equation by solving the following equation:

$-k^{2} M_{\infty}^{2} \phi_{11}^{1}-2 k M_{\infty}^{2} \phi_{x 1}^{1}+\left\{\left[1-M_{\infty}^{2}-(\gamma-1) M_{\infty}^{2} \phi_{x}^{0}\right] \phi_{x}^{1}\right\}_{x}+\phi_{y y}^{1}+\phi_{z z}^{1}=0$

Note that the steady potential $\phi^{0}$, is required in the above equation. In practice, $\phi^{0}$ can be obtained from other theoretical formulations or derived from experimental data. Hounjet [74] is representative of this approach to unsteady transonic flow modeling.

\subsection{Computational Grid Effects}

Grid generation for unsteady problems in which the body boundary moves, such as for an oscillating control surface or an aeroelastic deformation, raises new issues over those involved in steady flows. At the TSD equation level, wherein the boundary conditions are applied on a nonmoving, mean surface plane, stationary Cartesian grids have been used. For unsteady problems, care must be taken to ensure the fidelity of signals propagated through the stretched grids which are used. Seidel et al. [140] and Bland [34] give results for the TSD equation. A key effect of grid stretching is to modify the "dynamic impedance" of the mesh at internal grid points, leading to "internal reflections" of waves which may return to the vicinity of the modeled aircraft components and contaminate unsteady solutions. It is shown that this issue is typically more severe for exponentially stretched meshes, frequently used for steady calculations, than for meshes with less severe stretching in the near field. This effect is alleviated in three-dimensional flows. Bland [35] provides guidelines for generating grids and selecting time-step size for accurate unsteady computational characteristics. Finally, this effect has not been observed or documented for calculations utilizing the higher level Euler or Navier-Stokes equations.

For flow modeling equations higher than the TSD equation, the body-conforming grids used must be realigned with the moving body at each time step to maintain accuracy. Schemes for accomplishing this have been studied as well as the necessity of moving the grid at all. When body motions are small with perturbations mainly normal to the surface, imposing "transpiration" boundary conditions on the mean surface location may be an acceptable approximation (Sankar et al. [137]). Steger [147] formulated the TLNS equations including terms accounting for grid motion. Steger and Bailey [148] used simple shearing of the grid coordinate normal to the surface to allow the grid to follow aileron motions. Chyu and his coworkers $[42,43]$ used an interpolation scheme for defining grids at intermediate steps between the extremes of motion for oscillating airfoils and for fixed outer computational boundaries. Anderson et al. [3] present EE results for dynamically moving airfoils and wings in which the entire mesh is rotated to follow rigid airfoil and wing motions. For aeroelastic motions of flexible structures more general methods for dynamically moving the mesh are required. Guruswamy [67], Ide and Shankar [84], and Nakamichi [115] describe methods wherein the curvilinear coordinate normal to the surface is sheared based upon the instantaneous surface normal displacement as computed by the time-marching aeroelastic equations.

The above methods have all been implemented using structured grid meshes in which computed variables for neighboring grid points are stored in adjacent computer memory cells. Unstructured grids, which can be implemented with triangular grid cells in two-dimensions and as tetrahedral cells in threedimensions, offer more flexibility in modeling complex geometries. Batina has developed a method for moving such body conforming meshes to maintain alignment during aeroelastic motions for airfoils [24] and complete configurations [23]. A network of springs is associated with the mesh in which the edge of each mesh cell is represented by a spring whose stiffness is related to the length of the edge. At each time step, the new location of the body boundary obtained from the aeroelastic equations of motion is used to solve for the new static equilibrium location of the nodes of the spring network. Robinson et al. [130] modified this grid motion technique for structured grids and give results of wing flutter calculations using an Euler code. Rausch et al. [124] further refined the method, treating spatial grid cell adaption (mesh enriching and coarsening procedures) on unstructured meshes.

\section{Computational Aeroelasticity Resource Assessment}

In this section, estimates of computer resources necessary to produce accurate, converged results are given. This is done prior to the discussion of the detailed applications in the following sections in order to provide a framework within which to assess what has been accomplished and where further work is necessary.

The measure which will be used for computational aeroelasticity resource requirements is the Computer Processing Unit (CPU) run time for a single steady or dynamic time-marching calculation. Typically, a steady calculation is required to establish an initial flowfield for subsequent dynamic calculations. Each dynamic calculation produces a time history of aeroelastic response from which stability or response measures, such as modal frequency and damping, may be derived. The CPU run time for a CFD calculation can be estimated from the relation

$$
T_{c p u}=N_{c y} N_{s t / c y} N_{g p} \tau
$$

relating computer CPU run time, $T_{c p u}$, to the number of computational steps, $N_{s t}$; the number of cycles of oscillation for a given frequency, $N_{c y}$; the number of steps per cycle (required

for accuracy or stability), $N_{s t / c y}$; the number of grid points, $N_{g p} ;$ and the algorithm speed, $\tau$.

The algorithm speed, $\tau$, is a common measure of the speed of an algorithm given in microseconds per grid point per time step $(\mu \mathrm{sec} / \mathrm{gp} / \mathrm{st})$. Values used for this parameter herein assume machine speeds typical of a Cray 2 class supercomputer: 250 million floating point operations per second. Lower values of $\tau$ are associated with less complex algorithms, such as explicit methods, while more complex algorithms yield larger values. However, the higher level algorithms (e. g. implicit, upwindbiased, etc.) allow larger time steps and are generally favored 


\title{
Table II COMPUTATIONAL RESOURCE ESTIMATES FOR HIGH SPEED, LOW ANGLE CONDITIONS
}

\author{
Single half-span wing; up to "locally separating" flow
}

$$
T_{c p u}=N_{c y} N_{s t / c y} N_{g p} \tau
$$

\begin{tabular}{|c|c|c|c|c|c|}
\hline & & TSD & TSD-VISC & Euler & TLNS \\
\hline $\begin{array}{l}\text { Algorithm speed, } \\
\mu \mathrm{sec} / \mathrm{gp} / \mathrm{st}\end{array}$ & $\tau$ & 3.0 & 6.2 & 60 & 65 \\
\hline $\begin{array}{l}\text { Number of steps } \\
\text { Steady state } \\
\text { Dynamic }\end{array}$ & $\begin{array}{l}\mathrm{N}_{\mathrm{st}} \\
\mathrm{N}_{\mathrm{cy}} \\
\mathrm{N}_{\mathrm{st} / \mathrm{cy}}\end{array}$ & $\begin{array}{l}1000 \\
3-7 \\
100-300\end{array}$ & $\begin{array}{l}1000 \\
3-7 \\
100-300\end{array}$ & $\begin{array}{l}1000 \\
3-7 \\
300\end{array}$ & $\begin{array}{l}5000 \\
3-7 \\
1000\end{array}$ \\
\hline Number of grid pts & $\mathrm{N}_{\mathrm{gp}}$ & $200 \mathrm{~K}$ & $400 \mathrm{~K}$ & $240 \mathrm{~K}$ & $650 \mathrm{~K}$ \\
\hline $\begin{array}{l}\text { Run time, hrs } \\
\text { Steady } \\
\text { Dynamic }\end{array}$ & $\mathrm{T}_{\text {cpu }}$ & $\begin{array}{l}0.2 \\
0.05\end{array}$ & $\begin{array}{l}0.7 \\
0.2-1.4\end{array}$ & \begin{tabular}{|l|}
4.0 \\
$3.6-8.4$
\end{tabular} & $\begin{array}{l}59 * \\
35-82^{*}\end{array}$ \\
\hline
\end{tabular}

*Assumes uniform global time step; no zonal decomposition

for calculations requiring time-accuracy. For a TSD code, values are in the range of $3-10 \mu \mathrm{sec} / \mathrm{gp} / \mathrm{st}$ while Navier-Stokes codes call for values ranging from $10-100 \mu \mathrm{sec} / \mathrm{gp} / \mathrm{st}$. A value of 65 will be used below for the Navier-Stokes code resource estimates. This is representative of the speed for a threedimensional implicit, upwind-biased code.

Table II presents computational resource estimates for a single steady or unsteady (aeroelastic) response calculation for a high speed, low-angle problem. This implies that attached or only mildly separated flows are to be treated. The estimates are for a single half-span wing panel which is typically utilized when symmetry may be assumed. The four classes of CFD codes listed are: inviscid TSD, TSD with interacted boundary layer model (TSD-VISC), Euler equations, and Thin-Layer NavierStokes equations. The number of steps for steady and dynamic response calculations are typical of those reported in the literature. The number of grid points have been selected based upon published grid convergence studies: see Anderson and Batina [4] for TSD and EE results and Vatsa and Wedan [157] for Euler and Navier-Stokes results. The second and fourth columns giving estimates including viscous modeling are the most interesting as this level of flow modeling is required in order to achieve desired levels of improvement over well developed linear unsteady aerodynamic methods. The estimates range from approximately one hour per case for the TSD-VISC capability to over 50 hours for codes based on the TLNS equations. Treatment of full-span configurations would double these estimates and increased geometric complexity, such as additional lifting panels and bodies which would be called for in complete configuration modeling, would also increase these estimates. Each

additional component modeled (tail, fuselage, etc.) would cost roughly the amounts given in the Table for the single wing. This last cost factor is due to the nature of grid point densities used, wherein the large majority of grid points are clustered near the body surfaces with the grid density coarsening quickly away from surfaces. It is noted that the TLNS estimate is likely to be conservative in that a uniform global time step (limited by small grid cells in boundary layers) is assumed. A number of straightforward modeling changes could lead to more economical methods with no loss of accuracy.
Higher-angle vortex-dominated flows call for significantly more complicated flow modeling: flow separation over the forebody/wing must be accurately predicted; vortex formation and the convection of vorticity over significant distances without loss of accuracy are involved. Perhaps the most difficult computational aeroelasticity challenge is vertical tail buffeting wherein vortical flow from the region of the forebody/wing is convected downstream, possibly encountering vortex bursting, and impacts on the flexible tail structure. CFD computations of these features are beginning to emerge and are reviewed below. Edwards [55, 56] assesses the computer resource requirements for such calculations along the lines given above. The flowfield is assumed decomposed into three regions: an inner viscous region adjacent to bodies and lifting surfaces, a nearfield region encompassing the vehicle and the vortical flow region above it and an outer inviscid, irrotational region. Assuming these three regions to be modeled using TLNS, Euler and potential equations respectively leads to the following rough computer resource estimate for a tail buffeting calculation:

$T_{c p u}=N_{c y} N_{s t / c y} \frac{f_{\max }}{f_{\min }} \tau\left[196,000 N_{\text {bod }}+\frac{V_{I I} N_{g p / w l}^{3} f_{\max }^{3}}{U_{\infty}^{3}}+50,000\right]$

The frequency bandwidth of interest is specified by $f_{\min }$ and $f_{\text {max }}, N_{\text {bod }}$ is the number of lifting surfaces and bodies wetted by viscous flow, $V_{I I}$ is the volume of the nearfield vortical flow region and $N_{g p / w l}$ is the number of grid points per spatial wavelength assumed necessary for accurate vortical flow calculations. The sample estimate given by Edwards indicates run times on the order of 1000 hours for such a calculation. Use of an Euler code for a similar calculation leads to reduced estimates on the order of 100 hour run times. However, issues related to the use of inviscid Euler codes for this type of calculation indicate caution in their use [55].

\section{Low-Angle, High-Speed Flow Applications}

This section will discuss applications of CFD methods for flows which are generally at lower angles and high speeds. First, the available experimental databases needed for validation of CFD codes are described. Then, recent applications of 
CFD methods for unsteady transonic aerodynamic analysis are presented, followed by a survey of aeroelastic applications for flutter prediction. Finally, the status of complete aircraft configuration modeling is illustrated through cited examples found in the current literature.

\subsection{Experimental Data Bases for CFD Code Validation}

The AGARD Structures and Materials Panel Subcommittee on Aeroelasticity has selected experimental unsteady pressure data sets as Standard Configurations to provide reference computational test cases for CFD code validation. Data from essentially rigid models oscillating in selected degrees of freedom is available for six airfoils (Bland [30]) and five wings (Bland [31]). Reference [11] contains many comparisons with these data sets. Edwards and Thomas [52] survey calculations for these and other cases, providing tables of example applications. Detailed comparisons of calculations with these experimental data sets can be found in Angelini et al. [5], Houwink [77], Bland and Seidel [32], and Howlett and Bland [81].

The major intended use of unsteady aerodynamic calculations is for prediction of aeroelastic response of aircraft and, more specifically, flutter. There have been numerous published calculations of two degree-of-freedom airfoil studies which are devoid of experimental data comparisons since realistic 2-D flutter models are very difficult to fabricate. On the other hand, there are only a small number of published 3-D flutter calculations which are compared with experimental results. An important reason for this is the detail and effor required to perform a valid flutter analysis of a flutter model. Vibration mode shapes and masses must be accurately calculated or measured and surface coordinates measured.

In many instances, experimental flutter data obtained for actual or proposed flight vehicles are considered proprietary

by individual private organizations, and consequently, are not in the public domain. However, Yates [166] describes an AGARD standard aeroelastic flutter model consisting of a 45 degree sweptback wing for which extensive flutter test results are available. Also, NASA Langley Research Center has recently begun a multi-year experimental program to generate extensive flutter and unsteady aerodynamic data suitable for aeroelastic CFD code validation applications. This research activity, known as the Benchmark Models program, is outlined in Bennett et al. [27]. Rivera et al. [126] describe the first flutter and unsteady pressure measurement model test completed as part of this ambitious test program.

\subsection{Unsteady Aerodynamic Applications}

7.2.1 Linear Aerodynamic Methods. Although the major focus of this survey is on computational methods for nonlinear unsteady flows, it is prudent to be aware of the capabilities of linear methods for two reasons. First, economy of effort demands that proof be offered that supposedly more accurate methods do indeed make a difference. Secondly, new methods should always be checked against older well calibrated methods for conditions at which both should produce similar results, thus protecting against inappropriate use of "higher-order" methods.

For subsonic lifting surfaces, the standard linear methods are the doublet-lattice method (e.g. Rodden et al. [131] and the kernal function method (e.g. Rowe and Cunningham [133]).
Recent developments of unsteady supersonic aerodynamic theories have been the potential gradient method of Hounjet [76] and the harmonic gradient method of Chen and Liu [40, 41]. These references contain numerous examples of aeroelastic applications.

These linear aerodynamic methods have been highly refined and calibrated with aeroelastic model test data. Coupled with powerful finite element structural dynamic analysis programs, they are used at all stages of design. Two notable analysis codes, NASTRAN and Elfini, are described by Johnson and Reymond [89] and Petiau and Brun [121].

Liu et al. [104] describe extensions to an unsteady aerodynamics panel method to permit more accurate modeling of wing/fuselage configurations for subsonic flow field conditions.

Improvements to the technique used to implement unsteady boundary conditions on the body surfaces, the addition of an embedded singularity to simulate the aft flow separation in the body wake region, and the use of constant-pressure wing panels (as opposed to doublet lattice panels) are discussed in the paper. Results are presented for wing-tail, body-alone, and wing/body configurations.

7.2.2 Nonlinear Aerodynamic Methods. Much effor has been directed towards predicting unsteady transonic aerodynamic flows using the transonic small disturbance equation. The XTRAN3S TSD code has been extended to allow treatment of wing-tail (Batina [18]) and wing-fuselage (Batina [19] and Guruswamy and Goorjian [70]) configurations. Batina [20] has adapted an approximate factorization (AF) solution algorithm based upon the work of Shankar et al. [142] to the TSD potential equation. The AF algorithm is implemented in a computer code termed CAP-TSD (Computational Aeroelasticity Program - Transonic Small Disturbance) developed at NASA Langley Research Center (Batina et al. [21]). The code permits the aeroelastic analysis of complete aircraft through the modeling of multiple lifting surfaces and bodies. Results are presented for five configurations illustrating this capability. Steady and unsteady pressures for the F-16C aircraft modeled by four lifting surfaces and two bodies are presented and agreement with experimental steady pressures is considered good. The grid used for these calculations contained 324,000 points. The calculations required $0.88 \mathrm{CPU}$ seconds per time step or 2.7 microseconds per grid point per time step on the CDC VPS-32 computer. Thirteen million words of memory was required. Pitt et al. [123] give flutter analyses obtained with the XTRAN3S code and the CAP-TSD code. Both codes were used for the wingalone analysis of the F-15 and F/A-18 aircraft. The latter code was used to study canard/wing/tail and wing/launcher/tip missile configurations. There is a general lack of unsteady experimental data for complex configurations with which to validate such computations.

Rodman, Nixon and Huttsell [132] describe modifications to the XTRAN3S code to permit the use of experimental steadyflow pressure data in the unsteady flow solution of the TSD equation. The TSD equation is split into a steady and an unsteady component. Experimental surface pressure data are used to define a "strained-coordinate" system, which is then used to solve the perturbation equation for the unsteady flowfield potential. The method is limited to those situations in which shockwaves are preserved throughout the unsteady motion. Test cases are presented for both 2D and 3D configurations (NACA 
64A006, NLR 2302, airfoils and the F-5 wing).

Angelini, et al. [5] report on a large number of unsteady transonic-flow computations performed using five different CFD methods. The computational procedures include a 1) 2D TSD code, 2) a 2D coupled TSD code/boundary-layer method for unseparated turbulent flows, 3) a 2D coupled TSD/boundary-layer code for unseparated and separated laminar and turbulent flows, 4) a 2D Euler code for inviscid flows, and 5) a 3D TSD code for inviscid flows. Computed results are presented for several AGARD standard airfoil configurations and a rectangular wing model. Some comparisons are made with available experimental data.

Sotomayer, Sankar, and Malone [146] provide a comparison of computed results for the F-5 wing using three different numerical procedures. Results are presented for a transonic small disturbance code (XTRAN3S), a full potential code (USIPWING), and an Euler code (GTEUL-3D). Steady and unsteady-flow results are compared with experimental data for several Mach numbers $\left(M_{\infty}=.8, .9, .95, .99\right.$ and 1.35). Sotomayer and Borland [145] describe an application of the XTRAN3S code to the prediction of unsteady wing airloads arising from oscillating control surfaces. The boundary conditions used to simulate unsteady control surface motions are outlined. Numerical results for the F-5 wing with a deflected and oscillating trailing-edge flap are presented for a range of transonic Mach numbers. Comparisons are also made with existing experimental data for this wing.

A number of investigators have also used the FPE as a basis for developing unsteady transonic flow solution procedures. Isogai [86] describes a procedure to solve the quasi-linear form using a finite difference procedure on a stretched Cartesian mesh. A quasi-conservative form of Jameson's rotated differencing scheme is used to capture shock waves. Since the grid used in this method is not boundary conforming, interpolation formulas are used to project values of the surface boundary conditions to appropriate computational grid points near the actual body surface. A semi-implicit solution procedure is used to advance the resulting equations in time. Computed results and experimental data are presented for the ONERA M6 wing (steady flow) and the NORA wing (steady and unsteady flow).

Sankar and Malone [136] describe two different procedures (SUNTANS and USIPWING) used to solve 2D and 3D problems, respectively. These methods differ in the manner in which surface motion is prescribed. In the SUNTANS code [109] the computational grid deforms with time to permit an exact implementation of numerical boundary conditions at the instantaneous airfoil surface. In the USIPWING code [135], a surface transpiration technique is used to simulate small unsteady motions of the wing surface. Reference [136] presents 2D and 3D computed results compared to experimental data for a number of airfoil and wing geometries.

Additional 2D FPE methods are presented by Schippers [138] and Whitlow [163]. Schippers describes the mathematical formulation and numerical implementation found in the NLR TULIPS code. The TULIPS code solves the $2 \mathrm{D}$ full-potential equation in strong-conservation form. The FPE is solved on a body-fitted grid, using a second order time-integration procedure, together with flux-splitting techniques. The resulting finite-difference equations are integrated in time using the implicit method of fractional-steps. The algorithm is demonstrated for unsteady transonic flows about the NACA 64A010 aiffoil and an airfoil of the ONERA M6 wing. Whitlow describes modifications to a 2D Full Potential equation solution procedure which simulate nonisentropic flow effects arising from strong transonic shockwaves. A nonisentropic formulation is used to modify the density upwind biasing incorporated in the original FPE code. Calculated results are presented for several airfoil shapes.

The important effects of control surface effectiveness and hinge moments are studied in Bharadvaj [29] and Ominsky and Ide [119]. Both studies involve full potential equation codes and the former includes the effect of a 2-D quasi-steady boundary layer model to calculate pressures due to oscillatory control surfaces on a high aspect-ratio supercritical wing. The latter reference studies multiple surface control of a free-to-roll model for simultaneous flutter suppression and roll control.

Damodaran [45], Whitfield et al. [162], Chaderjian and Guruswamy [39], and Nakamichi [115] provide four examples of the use of structured-grid Euler/Navier-Stokes solvers for unsteady aerodynamic applications. Damodaran describes an application of Jameson's explicit Runge-Kutta time-stepping scheme to the solution of the 2D Euler equations for unsteady transonic flows about oscillating airfoil configurations. Nonreflecting far-field boundary conditions are used to help reduce non-physical wave reflections at the outer boundary of the computational grid. Numerical results are presented for the NACA 64A010 and the NACA 0012 airfoils. Additional calculations are presented for an airfoil experiencing axial oscillations (simulating a helicopter-rotor environment) and for the transient aerodynamic response of an airfoil due to angle of attack change. For the latter case, comparisons are made with classical linear aerodynamic theory. In Ref. [162], the authors describe a solution procedure for the 3D Euler equations in transonic flow. Dynamic multi-blocked grids are used to model complex aircraft configurations. The Euler equations are cast in strong conservative form and solved using an implicit, approximately factored scheme. Computational results are presented for a wing with store and a wing/counter-rotating prop-fan configuration. Limited comparisons with experimental data are provided for the wing/store configuration. Reference [39] describes a computational procedure for the thin-layer Navier-Stokes equations. The method can be used to model complex configurations via the multi-block technique for grid-generation. An implicit approximate-factorization diagonal algorithm is used to integrate the fluid dynamics equations. The method is considered first-order accurate in time. Computational results are presented for a low-aspect-ratio rectangular wing. Both steadyflow and unsteady-flow calculations are compared, and one case is presented for which the wing grid is split into an inner and an outer region in order to demonstrate the time accuracy of the method. Finally, Nakamichi presents comparisons of TLNS code results for the NORA wing, one of the AGARD Standard Configurations. A moving grid capability is employed, allowing the computational grid to follow the harmonically pitching wing motion.

Batina [23] has developed an Euler code based upon an unstructured grid with very general configuration modeling capability. Results are given for a supersonic fighter configuration with canard/fuselage/cranked delta-wing/tail/flow-through nacelle components modeled. Calculations are given for the complete vehicle oscillating in an aeroelastic mode and utilizing the moving mesh technique described above. Such unstruc- 
tured grid methods involve more complex programming than structured grid methods. Efficiency can be regained by algorithm improvements such as the temporal-adaptive algorithm described by Kleb et al. [93] which may relax the stringent requirement of using a global time-step dictated by the size of the smallest grid cells. See also Angelini and Soize [6] for a two-dimensional small perturbation treatment of body-fitted unstructured grids for unsteady calculations.

Mortchelewitcz and Sens [111] also describe a solution procedure for the 3D Euler equations using unstructured grids. An explicit-implicit two phase integration scheme is used to solve the discretized equations on a mesh of tetrahedral cells. Surface transpiration is used to simulate motion of the solid surfaces for unsteady computations. Sample computations are presented for a wing-body configuration and compared to experimental data. The experimentally measured wing deformation is included in the computational mesh to account for static aeroelastic effects.

7.2.3 Hybrid Aerodynamic Methods. A number of hybrid computational schemes have been developed which combine features of different aerodynamic theories. Liu and Kuo [102, 103] describe a Transonic Equivalent Strip (TES) method for computing unsteady transonic flow about three-dimensional wing configurations. The method uses 2D steady-flow pressure distributions to determine an "equivalent" airfoil shape obtained by an automated airfoil design procedure. This insures that the mean-flow transonic effects are matched closely. Then the equivalent shape is used to compute 2D unsteady transonic-flow using a nonlinear method, such as the LTRAN2 TSD code. Finally, the 2D unsteady transonic airloads are applied at each span station of the wing, using phase-lag modifications obtained from a 3D linear wave theory. Computed unsteady pressure distributions are given for several wing configurations, including wings with oscillating control surfaces.

Hounjer [74] describes a hybrid field-panel/finite-difference procedure (FTRAN3) which combines features of both linear and nonlinear methods. The field panel procedure is used to compute a proper radiating boundary condition on the outer boundary of the grid used for the finite-difference method. This permits the grid extent to be reduced in size, resulting in a decrease in computational effort overall. Hounjet implements a multigrid technique to further improve convergence of the finite-difference method.

Voss [160] describes other hybrid schemes. An unsteady field panel method (PTRAN3) for solving the time linearized 3D Transonic Small Disturbance equation is described. The wing and wake surfaces are modeled with unsteady dipole distributions, while the region near the wing surface, where compressibility effects are greatest, is modeled using volume sources. Voss also describes a combined field-panel/finite-difference procedure.

Zimmerman and Vogel [169] describe a time-linearized method for solving the transonic small perturbation equation (LIN TSP). Comparisons are made for the unsteady transonic airloads computed using several different methods, including LIN TSP, TSP and doublet lattice. Some calculations are made using both a steady and a quasi-steady boundary layer. The paper also investigates the behavior of higher order harmonics of the computed airloads, and concludes that for small amplitude motions the higher-order harmonics can be neglected.
Steiginga and Houwink [149] describe an engineering type method (Q3D) which couples 2D linear theory, 2D nonlinear theory and 3D linear theory, in order to predict unsteady transonic airloads on transport-type aircraft wings. In this method, 2D scaling matrices are first obtained by forming a ratio of unsteady 2D nonlinear to 2D linear AICs, where each nonlinear AIC is computed for a representative airfoil section of the 3D wing. The resulting scaling matrices are used to modify $3 \mathrm{D}$ AICs obtained from a doublet lattice analysis.

Finally, Reisenthal and Nixon [125] have supplemented the TSD equation with a transport equation for streamwise vorticity and a vector potential equation to predict vortex effects over missile configurations. Results for a complete missile configuration at subsonic, transonic, and supersonic speeds are encouraging.

\subsection{Aeroelastic Applications}

A restricted selection of the many publications of 2-Dpotential fow flutter calculations includes Isogai [85], Edwards et al. [51], Bland and Edwards [33], Berry et al. [28], and Zimmerman [169]. References [33] and [51] document the somewhat surprising "locally linear" nature of transonic potential flows. That is, about the steady mean flow condition which is a nonlinear function of Mach number and angle-of-attack, unsteady perturbation airloads behave very linearly for reasonable airfoil motions. Reference [51] included the static twisting of the aifoil due to the steady pitching moment into the flutter problem and demonstrated a marked effect upon flutter boundaries, particularly for the supercritical MBB-A3 airfoil. Reference [28] documented the utility of s-plane Pade curve fits of transonic airloads (which rely on the concepts of linearity and superposition) for aeroelastic analysis. Viscous effects are shown to generally result in larger values of flutter speed since transonic effects are alleviated by the boundary layer. Zimmerman [170] describes the application of several unsteady aerodynamic theories to the prediction of transonic flutter instabilities for a 2D airfoil profile. Flutter predictions are made using TSP theory, linearized TSP, TSP with quasi-unsteady boundary-layer and doublet lattice. Both frequency domain and time-marching methods are used in these flutter predictions.

Wu et al. [164] describe the use of a compressible-flow, FNS code for the prediction of unsteady airloads and flutter of 2D airfoil sections. Unsteady flow results are shown for harmonically oscillating NACA 0006 and NACA 0012 aifoils. Time marching aeroelastic solutions are given for the NACA 0012 airfoil showing stall flutter for several different Mach numbers and Reynolds numbers.

At the NLR, a number of unsteady aerodynamic computational procedures have been developed for use in transonic flutter prediction (Hounjet [74] and Steiginga and Houwink [149]). Zwann [168] describes an investigation into the accuracy of several of these methods for predicting flutter of a transporttype wing model. The methods examined include doublet lattice with quasi-steady corrections, the Q3D quasi 3D method and the FTRAN3 hybrid field-panel/finite-difference procedure. Corrected doublet lattice results are shown to display a flutter dip, probably due to the use of available experimental sectional airloads data. The flutter dip computed is, however, unconservative. The correlations of flutter speed versus Mach num- 
ber reported for the Q3D code are slightly conservative, but in good agreement with test results over a range of Mach numbers, and up to a point where the flow begins to separate. Several FTRAN3 predicted flutter points compared closely to the Q3D results. Hounjet and Meijer [75] give additional applications of the time linearized FTRAN3 code to flutter calculations for a fighter configuration with stores.

Other time-linearized finite-difference methods for solving the unsteady transonic flow about harmonically oscillating wings are described by Weatherill and Ehlers [161] and Shieh, Schoen, and Fung [143]. In Reference [161] the transonic small disturbance equation is split into a steady and an unsteady flow component, and the unsteady portion is solved using an out-of-core LU decomposition procedure (OPTRAN3). Sample flutter analysis results are presented for a flexible rectangular-wing. Reference [143] describes an implicit finite-difference procedure for solving the 3D full-potential equation for small unsteady perturbations of the body surface. The potential function is split into steady and unsteady components. The steady component can be obtained from a number of sources, such as higher order fluid dynamic equations (e.g. Euler, Navier-Stokes) or experimental data. Corrections are applied for nonisentropic effects, and the body motion is enforced using surface transpiration on a static grid conforming to the mean position of the solid surfaces (ZUNAS code). Computed results are given for unsteady oscillations of a NACA 0012 airfoil and for flutter boundaries of a $3 \mathrm{D}$ transport-type wing.

Comparisons of calculated and experimental flutter boundaries for wings have been given by Guruswamy and Goorjian [66], Isogai [87], Isogai and Suetsugu [88] and Myers et al. [113]. Isogai [85] studied the supercritical wing of Farmer et al. [57] using a nonconservative full potential code and an interacted boundary layer model. The trend of the transonic fiutter dip is very nicely predicted although the dip occurs about 0.08 low in Mach number. The premature flutter dip and the subsequent premature rise of the calculated boundary at higher speeds is of concern. Myers [113] also shows such a premature rise in the boundary. Finally, Isogai [87] shows flutter comparisons for a different supercritical transport wing which agree nicely with the experimental flutter dip.

Cunningham et al. [44] describe TSD code flutter calculations for the AGARD Standard Aeroelastic flutter model configuration [166]. This is a $45^{\circ}$ sweptback wing with a taper ratio of 0.66 . Experimental and computed futter boundaries are given for Mach numbers from 0.338 to 1.14 . For this 4 percent thick wing, transonic effects are delayed to high subsonic Mach numbers and linear theory results from both CAP-TSD and a kernel function program are in very good agreement with experiment up to $M_{\infty}=0.98$. Nonlinear CAP-TSD subsonic flutter calculations agree better with experiment than the linear theory, particularly for the change in slope of the flutter boundary near $M_{\infty}=0.95$. Robinson et al. [130] have also performed flutter calculations for this wing using an Euler code. The moving mesh scheme described above was modified for use with a structured grid algorithm and the flutter boundary prediction was in good agreement with the TSD code and experiment.

Borland and Nagaraja [37] describe extensions to the XTRAN3S code to permit aerodynamic modeling of wingpylon-stores and wing-fuselage configurations as well as the addition of supersonic analysis capability, non-reflecting bound- ary conditions and a Fourier analysis option for unsteady surface pressures. A number of applications of the code are presented for steady- and unsteady-flow aerodynamics and static/dynamic aeroelastic solutions. The configurations studied included an A6 fighter configuration with stores, and a transport wing/fuselage configuration with winglet.

Mulak, Meurzec and Angelini [112] describe a finitedifference procedure to solve the $3 \mathrm{D}$ unsteady transonic small disturbance equation. The method solves a discretized form of the equation using an ADI splitting technique. Unsteady generalized airforces are computed for a number of normal modes for a swept, high-aspect-ratio transport-type wing. Some comparisons are made for steady and unsteady surface pressures and test data at a transonic Mach number. Flutter calculations are performed using different wing twist distributions corresponding to jig and flight condition shapes. A comparison is also presented for flutter calculations using both the doublet lattice and the TSD method.

Wong and Lee [165] describe a numerical procedure to compute unsteady transonic flow about 3D isolated-wing configurations (UST3D code). The procedure solves the transonic small disturbance equation, split into a nonlinear steady component, and a linear unsteady component. The steady part of the equation is solved with an implicit Newton-iteration scheme, used in conjunction with a preconditioned gradient type procedure. The unsteady part is solved with a semi-implicit technique which is explicit in the stream direction and implicit in the cross-flow planes. A nonreflecting far-field boundary condition is applied at the outer boundaries of the Cartesian mesh. Computed results are given for a fighter wing. Real and imaginary components of lifting pressure are given for a Mach number of 0.9 and $\mathrm{k}=$ 0.45 . Aeroelastic results are compared with doublet lattice computations for unsteady pressures and a sample flutter calculation is presented.

Five additional applications of TSD codes to wing flutter calculations are described in Ref. [13], Bennett and Batina [26], Gibbons et al. [62], Silva and Bennett [144], and Guruswamy and Goorjian [69]. Reference [13] describes an implicit finitedifference procedure ( $\triangle$ TRANS code) for solving the 3D transonic small disturbance equations on a Cartesian mesh. Aeroelastic calculations are made for the Falcon 900 wing at transonic Mach numbers, both with and without the incorporation of static aeroelastic deformations. Comparisons are also made with a doublet lattice method at a subsonic Mach number of 0.8 . Bennett and Batina [26] present flutter calculations for a three percent thick clipped tip delta wing with a leading edge sweep angle of 50.5 degrees. The Mach range covered was $0.6-0.9$. At the lower Mach numbers the results were in excellent agreement with linear theory, while there was a 6 percent reduction in flutter speed from linear theory at $M_{\infty}=0.907$, bringing the results into better agreement with experiment. Gibbons studied a 70 degree swept delta wing for Mach numbers ranging from 0.6 to 3.0. The calculated flutter speeds are in very good agreement with experiment at transonic speeds. At supersonic speeds, the trend of the flutter boundary with Mach number agrees well with experiment. Silva and Bennett [144] show transonic flutter boundary predictions for a complex wind tunnel model. The blended wing-fuselage and tip ballast store were modeled. Significant improvement in predicted flutter points over those calculated with linear theory are shown for high transonic Mach 
number experimental points. Finally, Guruswamy and Goorjian [69] give flutter boundary calculations for a rectangular wing which agree well with experiment including a low supersonic condition.

Several investigators have studied aifoil and wing limitcycle-oscillations (LCO) using both CFD-based and empiricallybased computational methods. Bendiksen and Kousen [25] and Kousen and Bendiksen $[94,95]$ studied nonlinear aeroelastic dynamic response of an airfoil section using a CFD method based on the Euler equations. The Euler procedure is a finitevolume scheme solved using an explicit five-step Runge-Kutta algorithm. A two-degree-of-freedom structural dynamics model was solved iteratively along with the fluid dynamic equations to predict the dynamic response of the airfoil section. Aeroelastic stability was studied for NACA 0006 and NACA 64A010 airfoil sections over a range of Mach numbers. Limit cycle oscillations were found to occur at transonic speeds as a bifurcation phenomenon. A divergence/flutter interaction [94] was identified for which the airfoil dynamically approaches a static equilibrium offset at non-zero angles-of-attack.

Kousen and Bendiksen [95] also examined the effects of torsional spring free-play on the flutter response of $2 \mathrm{D}$ airfoil sections restrained by a two-degree-of-freedom elastic system (pitch and plunge). For the 2D case, the incorporation of freeplay in the torsional spring lowers the stability boundary for conventional flutter. A bifurcation phenomenon is shown to exist above the maximum reduced velocity for flutter. Above these values, a limit-cycle behavior is exhibited by the airfoil/spring system. This $2 \mathrm{D}$ analysis method was applied to the flutter behavior of a well known typical section model of sweptback wing bending and torsion modes. It is shown that the dual-mode instability calculated for this model corresponds to a region, or pocket, of limit-cycle behavior. Finally, the effects of reduced airfoil thickness were examined and shown to be destabilizing, leading to limit-cycle behavior at lower Mach numbers than for a thicker airfoil section.

Meijer and Zwann [110] describe a semi-empirical computational method for the prediction of LCO occurring on fightertype aircraft (see figure 5). Steady-state experimental surface pressure data are used to compute generalized airforces required to solve the structural dynamic equations of motion for a vehicle. The surface pressures are tabulated versus Mach number and steady angle-of-attack and utilized during the transient response simulation in a quasi-steady fashion wherein the instantaneous, induced angle of attack is used to reference the pressures. Both rigid and elastic mode shapes are used in the computations. Sample calculations of dynamic structural responses were made for two vehicle configurations, an isolated wing and a wing-with-missile configuration. Limit-cycle oscillations were found only for the clean-wing configuration, where the computed frequency and mean angle-of-attack data compared well with flight test results. For the flight conditions were LCO was observed, single-degree-of-freedom predictions using single mode shapes failed to show indications of LCO behavior. The authors state that LCO may not be associated with single degree-of-freedom systems, but rather with multiple-degree-offreedom systems where modal coupling takes place, in a manner similar to the classical flutter mechanism.

The suppression of fiutter instabilities by active controls has been demonstrated using CFD methods by Ominsky and Ide
[119] and Guruswamy [68]. Ominsky and Ide use a Full Potential equation CFD method to evaluate aeroservoelastic control laws for flutter suppression. A test case for a swept wing is created by first determining a flutter condition to be controlled. A single trailing edge flap is deflected with time using a simple open loop multi-parameter control law. The parameters of flap amplitude of oscillation frequency and phase lag are studied to determine their effects on flutter response and a second-order control law is used in a closed loop manner to control the identified flutter instability. Guruswamy summarizes efforts at NASA-Ames to develop CFD methods for aeroelastic applications. Applications of the ATRAN3S code (TSD) and the TNS code (Euler/Navier-Stokes) are given. These include unsteady aerodynamic results, flutter analyses and flutter suppression demonstrations. Some comparisons with experimental data are given.

Schuster et al. [139] have developed a FNS code using zonal grid generation techniques to enable aeroelastic analysis of complete vehicles. Static aeroelastic calculations are presented for a fighter aircraft at a high wing-loading transonic condition. Calculated twisting of the aeroelastically tailored wing/fuselage configuration compares favorably with experiment.

Obayashi and Guruswamy [118] describe applications of the ENSAERO code to compute unsteady aerodynamics of a delta wing in oscillatory and ramp motions. The ENSAERO code is an Euler/Navier-Stokes method which includes a structural dynamics model to account for wing flexibility. The method solves the thin-layer Navier-Stokes equations and incorporates an algebraic turbulence model. Comparisons of theory and experimental data are presented for a number of steady flow and unsteady flow (pitching-oscillations) conditions. Computational results are also presented for a delta-wing undergoing an unsteady ramp motion (pitch). The computed results indicate a vortex breakdown condition occurring for a ramp motion which reaches a maximum amplitude of 10 degrees angle-of-attack.

Vinh et al. [159] present an interesting augmentation to the aeroelastic response capability of such codes. In addition to the structural dynamic equations of motion normally used for aeroelastic analysis, two additional degrees of freedom modeling the rigid aircraft short period mode were implemented along with an automatic trimming capability. This allows the interaction of the elastic modes upon vehicle stability and control to be calculated in a straightforward manner.

\subsection{Viscous-Inviscid Interaction Applications}

The transonic aeroelastic stability illustrations shown in figures 3-5 all involve strong viscous effects which inviscid analyses cannot predict. In order to achieve desired improved accuracy in predictive methods for such cases, an accounting of the effect of the viscous boundary layer is mandatory. Flows which must be treated include those with thickening boundary layers, incipient separation, shock-induced and trailing-edge separation, and alternately separating and reattaching flows. The computational resource estimates given in Table II indicate the severe cost incurred by going to higher level CFD flow models and lead to the desirability of achieving the maximum possible viscous flow analysis capability with lower level CFD methods coupled with interacted viscous boundary layer models. Due to the importance of this issue for aeroelastic analysis, this section focuses on such applications. Interacted boundary layer methods have been developed for unsteady applications using 
direct solution for attached flows and indirect solution for separated flows. Calculations using TSD, full potential, and Euler equation codes have been extensively reported.

Examples of viscous flow effects for attached 2-D flows (direct solution method) are given by Guruswamy and Goorjian [65], Howlett [80, 81], and Houwink [77, 78]. All of these references use an integral boundary layer model coupled to a TSD inviscid method and calculations for the AGARD Standard Configurations are given (NACA 64A010, NACA 64A006, MBB-A3, NLR 7301, and NACA 0012 airfoils). Pirzadeh and Whitfield [122] report a 3-D direct viscous solution coupled to the Euler equations and give results for the NACA 64A010 airfoil and the ONERA M6 wing.

Unsteady results using the quasi-simultaneous method coupled to TSD solvers are reported by Houwink and Veldman [79] for 2-D cases and by Henke et al. [71] for 3-D cases. Houwink gives separated flow results for an oscillating supercritical aiffoil and for an aiffoil with a deflected spoiler. Henke gives details of a similar viscous coupling method implemented in a stripwise fashion in a 3-D TSD code and including aeroelastic computations. Comparisons with measured unsteady pressure from a modern transport wing model with a supercritical airfoil are shown and flutter calculations using the interacted viscous-TSD code are compared with doublet lattice calculations.

The most elaborate viscous interaction calculations of unsteady separated 2-D flows have been published by LeBalleur and his coworkers $[98,99]$. An unsteady deflect integral boundary layer model is coupled to a TSD solver using the alternatingdirection implicit solution method which enables an incorporation of the strong interaction of the viscous influence during the final z-sweep. The semi-implicit solution procedure involves a relaxation solution of a viscous influence function to force convergence of the viscous and inviscid solutions at each time step. Reference [99] gives unsteady shock-induced separation results for an oscillating NACA $64 \mathrm{A010}$ airfoil, self-induced shock oscillations for a circular arc airfoil, and a supercritical airfoil with an oscillating spoiler. Giroudrouux-Lavigne and LeBalleur [63] further explore the self-excited shock oscillations, giving examples for the RA16 SCl supercritical airfoil and the NACA 0012 airfoil. The shock oscillation conditions are shown to coincide with experimentally observed buffet onset boundaries. It is interesting to note similar calculations of buffet onset boundaries reported by Hirose and Miwa [72]. Experimental buffet boundaries for the NACA 0012 and KORN 75-06-12 airfoils are compared with calculations from a 2-D TNS code. As in the above viscous-inviscid interaction results, self-excited oscillating shocks are observed for conditions in good agreement with the experimental buffet boundaries.

In summary, viscous interaction methods are capable of treating important transonic effects when coupled with lower level CFD methods. Impressive results have been published for 2-D flows. Similar capability for 3-D flows involving shock-induced separating and reattaching flows remains a most important research topic.

\subsection{Complete Aircraft Configurations}

This section will survey the most ambitious CFD modeling of complete aircraft in order to indicate the levels of details and the level of effort such studies involve. All of the cases cov- ered are for steady conditions; thus they can serve as a baseline for estimating resources which would be required for unsteady analyses. It is not surprising that the most detailed aircraft geometry modeling has been applied for cases at low angles of attack, near design conditions, where the flow is attached or mildly separated. These conditions are most important in design and the codes can be expected to perform at their best due to good flow quality (steady, attached, thin shear-layers, etc.). Thus, indications of the accuracy of results here will help to assess the readiness of the codes for the more demanding transonic flutter and low-speed, high-angle buffet conditions. Table III summarizes a number of CFD applications for such cases. All of these studies used the TLNS equations and all implemented simple turbulence models. All used convergence acceleration devices (local time-stepping, multigrid, etc.) which yield accurate results only for converged, steady flows. Except for Flores and Chaderjian [59], all made use of assumed symmetry in the flow to reduce by half the grid size (only one-half aircraft model analyzed). Finally, the codes used in these studies were generally second-order accurate in space and first-order accurate in time. Fujii and Obayashi [61] and Huband et al. [83] give results for complete aircraft models at transonic speeds while Thomas et al. [151] studies a detailed forebody/strake model at low speed and high angle. The latter is included due to its complex surface modeling. Vadyak and Schuster [156] give results for a sharp-edged generic fighter wing-body configuration at high angles. Kwon and Sankar [97] and Rizk and Gee [128]

give results of buffet flowfield calculations about two high performance fighter configurations at high angles. These last three cases of high angle vortex-dominated flows will be discussed in the next section.

Fujii and Obayashi [61] modeled the W-18 transport configuration as a wing-fuselage and made calculations for three angles-of-attack. The overall surface pressures compared fairly well with experiment except in the outboard wing region where elastic deformations were not accounted for. Their numerical algorithm allowed the calculations with $700 \mathrm{~K}$ grid points to be obtained in 5-6 cpu hours. Flores and Chaderjian's [59] study of the F-16A aircraft is one of the most ambitious applications 10 date. They modeled the wing, fuselage, tail, inlet, inletdiverter, and the exhaust nozzle using 27 grid zones for the half-airplane. The flow-through inlet was modeled including power effects. Comparisons with experimental pressures indicated that the wing leading-edge expansion was not adequately resolved and the wing shock location was off by $12 \%$ chord. Doubling the grid size to one million points allowed the calculation of the full-span aircraft at five degrees sideslip angle. This also doubled the runtime to 50 hours. With regard to buffet calculations, it is interesting to note that the vertical tail tip vortex for this condition dissipated within one tip chordlength due to numerical dissipation (due of grid stretching downstream of the tip). Huband et al. [83] studied the same F-16A (the inlet is faired over) for a low supersonic Mach number. Their fine grid solution $(1,241 \mathrm{~K}$ grid points) occupied 59 million words of memory and required 40 hours of runtime. They obtained favorable agreement with experimental surface pressures but the wing leading-edge suction peaks were not correctly predicted due to lack of numerical resolution. 
TABLE III - CFD APPLICATIONS TO COMPLEX AIRCRAFT GEOMETRIES

\begin{tabular}{|c|c|c|c|c|c|c|c|c|}
\hline Ref. & Configuration & $\mathbf{M}$ & $\alpha$, deg. & Re, $10^{6}$ & $N_{s t}$ & $N_{8 P}$ & $T, \mu \sec$ & $T_{\text {cpu, }}$ hrs \\
\hline Fujii ${ }^{61}$ & W-18 & 0.82 & $2,4,6$ & 1.67 & $3000+$ & $700 \mathrm{~K}$ & 9.5 & $5-6$ \\
\hline Flores 59 & F-16A w/inlet & 0.9 & 6 & 4.5 & 5000 & $500 \mathrm{~K}$ & $36+$ & 25 \\
\hline Huband $^{83}$ & $F-16 \mathrm{~A} w / 0$ inlet & 1.2 & 6 & 12.75 & $+0,000+$ & $1,241 \mathrm{~K}$ & $30+$ & 40 \\
\hline Thomas 151 & $\begin{array}{l}\text { F/A-18 forebody } \\
\text { \& LEX }\end{array}$ & 0.3 & 30 & $\begin{array}{l}0.74 \mathrm{WT} \\
10.0 \mathrm{FLT}\end{array}$ & & $300 \mathrm{~K}$ & & \\
\hline Vadjak $^{156}$ & generic wing/body & 0.3 & $\begin{array}{l}10,21 * \\
23,25\end{array}$ & 1.25 & 1.200 & $101 \mathrm{~K}$ & & \\
\hline Kwon ${ }^{97}$ & F-15 wing/body/inlet & 0.15 & $20^{*}$ & & & $224 \mathrm{~K}$ & & \\
\hline $\operatorname{Rizk}^{128}$ & F-18 aircraft & 0.243 & $30^{*}$ & 11.0 & 4,100 & & & 55 \\
\hline
\end{tabular}

*Unsteady vorrex flows

TABLE IV - CFD CALCULATIONS FOR HIGHLY-SWEPT WINGS AT HIGH ANGLES OF ATTACK

\begin{tabular}{|c|c|c|c|c|c|c|c|c|c|}
\hline Ref. & Configuration & $\Lambda$, deg. & $\alpha$, deg. & $\operatorname{Re}, 10^{6}$ & $M_{\infty}$ & $\mathrm{N}_{\mathrm{gP}}, \mathrm{K}$ & $\mathrm{N}_{\mathrm{st}}$ & $\tau, \mu \mathrm{sec}$ & $\mathrm{T}_{\mathrm{cpu}}, \mathrm{hrs}$ \\
\hline Hitzel $^{73}$ & $\begin{array}{l}\text { cropped delta, } \\
\mathrm{s,e}\end{array}$ & 65 & $\begin{array}{c}10,20,24,28^{*} \\
10-28,24^{*} \\
28 * 10\end{array}$ & 1.25 & $\begin{array}{l}0.4 \\
0.85 \\
1.20\end{array}$ & 270,540 & & & \\
\hline $\mathrm{Hsu}^{82}$ & delta, $\mathbf{r}$ & 60 & $12(20)$ & 1.3 & 0 & 712 & $700(1000)$ & 52 & $7(10)+$ \\
\hline $\mathrm{Hsu}^{82}$ & double-delta, r & $80-60$ & $12(20)$ & $\begin{array}{l}0.01 \\
1.3,100\end{array}$ & 0 & 955 & $700(1000)$ & 52 & $10(14)+$ \\
\hline $\mathrm{Hsu}^{82}$ & $\begin{array}{l}\text { cropped, } \\
\text { double-delta, r, }\end{array}$ & $80-40$ & $12(20)$ & 1.3 & 0 & 1021 & $700(1000)$ & 52 & $10(14)+$ \\
\hline $\mathrm{Hsu}^{82}$ & double-delta, $r$ & $80-60$ & $\begin{array}{c}6,12,15,20 \\
25,30,35^{*} \\
40^{*}\end{array}$ & 1.3 & 0 & 859 & $500(1000)$ & 52 & $6(12)+$ \\
\hline Fujii ${ }^{60}$ & double-delta, $\mathbf{r}$ & $80-60$ & $6,12,\left(30^{*}, 35^{*}\right)$ & 1.3 & 0.3 & 850 & $1000(5000)$ & 20 & $5(25)+$ \\
\hline Thomas $^{150}$ & delta, $s$ & 75 & $\begin{array}{c}0-40 \\
20.5,40^{*}\end{array}$ & 0.95 & 0.3 & 545 & 400 & 40 & 2.5 \\
\hline Krist $^{96}$ & delta, $s$ & 75 & 20.5 & 0.5 & 0.3 & $350(1000)$ & & & \\
\hline Agarwal $^{1}$ & delta, $s$ & 70 & $\begin{array}{c}(20,25,30, \\
35,40)^{*}\end{array}$ & 1.0 & 0.3 & & 1.2000 & & 3 \\
\hline
\end{tabular}

e-Euler code s-sharp leading-edge $\quad$ r-round leading-edge *-vorex burst/unsteady calculations t-estimated 


\section{High-Angle Vortex-Dominated Flow Applications}

Computational studies are beginning to delineate the required levels of effort to produce accurate computations for separated, vortex-dominated flows. In this section attention will be given to a brief assessment of the state-of-the-art in computing vortical flows using CFD methods, followed by a discussion of recent research efforts in two specialized categories, wing rock and vortex buffet.

\subsection{Status of CFD Prediction Methods for Vortical Flows}

Edwards [56] provides a detailed study of current research and applications into the use of CFD methods for the prediction of separated, vortical flows over airfoils, wings and bodies. Table IV lists a selection of these applications relevant to computational aeroelasticity at high angles. All except Hitzel [73] represent applications of the TLNS equation, while Hitzel utilizes the Euler equations. The major conclusions of this study are repeated here for completeness.

Calculations of stable vortex-dominated flows about simplified highly swept wing geometries at low speeds are available. Early Euler equation results gave encouragement to their use for such flows, but recent detailed studies point to issues regarding their accuracy and convergence for realistic geometries. The thin-layer Navier-Stokes equations, both laminar and turbulent are being used. The results capture key features of the flowfield such as primary and secondary vortices and surface pressure details. Indications that vortex breakdown is being simulated have been published. However, all cases surveyed indicated the need for further grid refinement in order to achieve quantitative agreement with experiment. Currently, grid sizes of 250,000 to $10^{6}$ grid points are being used for half airplane modeling leading to CPU runtimes of 2-25 hours. These grids are typical of those developed for attached shear flows with the highest grid density near the body. This leads to inadequate density in offthe-body regions where concentrated vortices are located and mesh enrichment methods are being developed to address this problem. A fundamental problem is the lack of any turbulence model designed for dynamic free shear layers.

Agrawal et al. [1] provide an interesting numerical study of this issue of off-the-surface turbulence modeling. Calculations for a sharp-edged delta wing from an Euler code, a laminar TNS code and a turbulent TNS code show vortex bursting at locations in general agreement with experiment. It is noted that the solutions are based on meshes that are not usually considered fine enough for resolving flowfields in primary vortex regions.

\subsection{Rolling Oscillations and Wing Rock}

Several studies have been published of unsteady airloads on rolling delta wings at high incidences. The dynamics of the leading-edge vortices which form at such conditions are one of the driving forces which can lead to wing-rock; a limited amplitude rolling oscillation which limits aircraft performance. Computations have been reported for low speed flows and for supersonic flows simulated via a conical flow assumption. Nayfeh et al. [116] use an unsteady vortex-lattice method to predict low-speed experimental wing-rock conditions.
Kandil and his coauthors have studied unsteady vortexdominated flow about delta wings using the conical flow assumption of supersonic flow. Reference [90] gives compressible Navier-Stokes results for rolling oscillations while Ref. [91] gives results for oscillating leading-edge flaps. Lee and Batina [101], again making use of the conical flow assumption, couple an unstructured grid Euler code with a wing rolling equation of motion to make calculations of supersonic wing-rocking motions. In a later work, Lee and Batina [100] demonstrated that this supersonic wing-rock phenomenon can be controlled with the use of oscillating, leading-edge flaps, activated by a simple control-law. More recently, Kandil and Salman [92] used an Euler conical flow solution procedure to study the control of wing-rock using leading edge flaps, together with a nonlinear control law.

\subsection{Buffet Flowfield Calculations}

Current and future military fighters are called upon to perform high angle maneuvers at elevated loading conditions and are experiencing structural fatigue problems due to dynamic buffet loads on aft components of the empennage, in particular the vertical tail(s). This has led to a desire for improved prediction methods for such flows, which emerging CFD methods might be expected to fulfill. Key flow modeling issues involve: i) unsteady flow separation for moderately swept, rounded leadingedge wings, ii) grid densities necessary for accurate calculation of vorticity convected over significant distances, iii) dynamic turbulence modeling for free shear layers, iv) detailed modeling necessary for accurate calculations of vortex instabilities, and v) vortex flows about wings at high subsonic speeds.

Three cases of the computation of buffet flowfields are given as the last three entries in Table III. All are for quite complex configuration models of high performance fighter aircraft and it has been noted above that this type of computation is the most demanding for aeroelastic. applications. Vadyak and Schuster [156] made calculations for a generic fighter configuration consisting of a sharp-edged strake/wing/fuselage. Low speed wind tunnel LDV flowfield data was available and crossflow velocity comparisons for $\alpha=21 \mathrm{deg}$. appear to be good. A bubble-type reverse flow region, indicating breakdown, is observed at approximately $x / L=0.9$. These comparisons for this high angle, vortex flow case are very noteworthy, particularly due to the complex geometry tested. However, the computational results, obtained with a grid of 101,000 points, cannot be regarded as converged.

Kwon and Sankar [97] give calculations for a half-span wing/body/inlet model of the F-15 aircraft. The FNS equations are solved using a hybrid time differencing scheme suitable for implementation on virtual memory machines. On a grid consisting of 224,000 points, calculations of unsteady, buffetlike flowfields are given for $M_{\infty}=0.15$ and $\alpha=20^{\circ}$. The unsteadiness is shown to consist primarily of a low frequency of about $5 \mathrm{~Hz}$. (full scale aircraft) and a high frequency ranging from $29-34 \mathrm{~Hz}$. The higher frequency content compares favorably with low speed wind tunnel tests for the complete aircraft (Triplett [154]) even with the relatively coarse grid used.

The final entry in Table III, that of Rizk and Gee [128], gives results for a complex configuration model of the halfspan F-18 aircraft. An overset zonal grid scheme consisting of ten grids was used to model the forebody, fuselage, LEX, 
faired-over inlet, wing, deflected leading-edge flaps, vertical and horizontal tails and an idealized boundary layer diverter vent. TLNS results are given for $M_{\infty}=0.243$ and $\alpha=30.3^{\circ}$. The solution required about 8 million words of memory and took about 55 hours of CPU time for 5,100 steps. Bursting of the LEX vortex in the vicinity observed in flight tests is shown. Unsteady loads on the vertical tail show a dominant frequency of $15-20 \mathrm{~Hz}$. Other higher frequencies are evident but are not well resolved. Triplett [154] gives test results indicating that the principal modal buffeting response frequencies of this aircraft are $15 \mathrm{~Hz}$. and $45 \mathrm{~Hz}$. Earlier calculations [127] indicate that finer meshes (e.g. about 2 million grid points) will be needed to accurately predict such features as the location of vortex bursting.

The buffeting response of launch vehicles is a critical issue in their performance. Up to the present, wind tunnel studies have largely been relied upon for the prediction of such airloads. Azevedo [14] presents novel TLNS results of buffeting response calculations for a hammerhead payload configuration.

In summary, ambitious applications of CFD to complicated aircraft geometries are being performed. Available studies are very encouraging in the overall agreement with experiment. Important areas are also being highlighted where additional grid resolution is needed to achieve local accuracy in such important features as suction peaks and shock locations. Computer runtimes for these cases are in the range of 5-55 hours (halfairplane). It is probable that accurate buffet calculations will require capability such as this forebody and wing flows in order to generate accurate "starting" conditions for the convecting vortex flows. It is also probable that similar capability will be required to calculate the buffeting response of the tail to the oncoming buffet flow. Indeed, this is likely to be a more difficult problem than the calculation of the "starting" wing flow due to the turbulent nature of the local flow at the tail.

\section{Discussion and Future Trends}

The proceeding sections have surveyed a segment of the large effors that have been spent on computational unsteady aerodynamics suitable for aeroelastic applications of fixed wing aircraft. These efforts can be divided into five general categories: i) development and demonstration of unsteady aerodynamic methods, ii) rigorous calibration and validation of these unsteady methods, iii) development and demonstration of aeroelastic analysis procedures, iv) rigorous calibration and validation of these aeroelastic procedures, and $v$ ) application of the resulting aeroelastic methods in the design or modification of actual or proposed flight vehicle configurations. Although nearly a decade and a half has passed since the earliest pioneering demonstrations of CFD applied to aeroelastic analyses, considerable efforts are still needed to reach a state where the aircraft industry, as a whole, accepts and routinely utilizes CFD for category v. activities. By far, the largest number of references cited herein fall within category i., and to a lesser extent, category iii. Also, to a large extent, the authorship of these cited references seems to indicate that a large segment of this research is currently occurring in universities and government sponsored research labora-

tories. Although there are some notable exceptions to this trend, many more industrial applications of aeroelastic CFD methods are needed and desirable. The following comments are offered regarding the current status and future trends of computational methods to meet the challenges listed in Table I and illustrated in figures 3-7:

1. Stability and control estimates, including aeroelastic effects, are being treated with linear methods which are well suited for design conditions well removed from flow separation boundaries where viscous effects are important. Initial demonstrations of the unified treatment of complete vehicle dynamics (rigid body and aeroelastic) using CFD methods are available.

2. Gust response analyses are currently conducted using linear aerodynamic methods. While CFD codes capable of treating complete configurations (necessary for gust response analysis) are available, there have been no reported attempts of such calculations other than isolated vortex-airfoil interactions.

3. A number of studies of flutter boundary calculations with CFD codes have been cited. Many more cases are needed in order to establish the required level of flow modeling detail which will provide accurate and useful results. It is not yet established for what conditions nonlinear flow modeling results differ sufficiently from linear results to justify their use. Also, the tradeoffs between the computational expense of the various levels of flow modeling (e.g. TSD-viscous vs. TLNS) and improved accuracy of results have not yet been established. A few cases have shown significant improvement over linear theory for small, but critical, ranges of transonic Mach numbers.

4. Prediction of these critical minimum transonic flutter speeds and nonclassical aeroelastic response phenomena, such as control surface buzz and aeroelastic limit cycle oscillations, certainly will require, at the very least, reliable, robust interacted boundary layer models capable of handling some amount of flow separation and reattachment. This capability is not yet mature for wings or more complete configuration modeling. This is also the case for the important design issues relating to aerodynamic control surfaces: control effectiveness and control hinge moments.

5. Cases of tail buffeting and structural fatigue being encountered by operational aircraft have focused attention upon this area. The achievements in the ability to compute vortexdominated flows are truly impressive and initial calculations of buffet-like flows appear to contain elements (frequency content, etc.) of realism. However, studies of the accuracy and convergence of such calculations in terms of grid density, sensitivity to initial conditions, repeatability, etc. are necessary. Two interesting questions regarding such flowfield simulations are:

a. if the flowfield simulations are repeatable, can the flow modeling contain adequate fluid dynamic modeling to ensure accurate buffet flows calculations?

b. if the flowfield simulations are very sensitive to initial conditions or are not repeatable, what measures are to be used in establishing the required number of cases and computational record lengths to ensure solution convergence? 
Regarding future trends in computational aeroelasticity the following comments are offered:

1. Robust interactive boundary layer methods coupled with all levels of inviscid codes will mature and be applied to aeroelastic stability and response calculations for high-speed, low angle conditions. The resulting improved accuracy in key areas such as transonic flutter prediction will be of great value.

2. Studies will delineate conditions best suited for the use of CFD methods. A hierarchy of the problem areas, keyed to the complexity of the flow problem being studied (e.g. Types I, II, or III flows), will be established and will be addressed by a suite of tools drawn from the alternative levels of CFD flow modeling.

3. Unstructured and hybrid grid methods will mature, leading to computations of quite detailed configuration complexity. The increased expense of unstructured grid methods (due to more complex coding) will be offset by the anticipated payoffs of the approach: fewer grid points required for comparable accuracy along with the complex geometry modeling capability. Continued improvements in algorithm efficiency will be seen for all gridding approaches; blockstructured, zonal and hybrid grid schemes will also be used in the search for robust, economical and user-friendly geometry modeling capability.

4. Improvements in conventional computer architectures will not achieve the orders of magnitude speedup needed to make the most complex aeroelastic computations, such as tail buffeting, routine. Massively parallel processing architectures may eventually provide relief, but not in the near future.

5. The need for experimental data bases specialized for the validation of computational methods for interesting aeroelastic flows is being addressed by NASA's Benchmark Models Program and the European Aeroelastic Models Program. Tests directed at unsteady off-the-surface flow measurements will continue to press the limits of instrumentation technology.

6. A final comment relates to differing uses of computational methods in the fields of aircraft performance analysis and aeroelastic analysis. The former application may be broadly typified as dealing with well-behaved, stable flows exemplified by performance and design studies where steady CFD methods are currently being utilized heavily. The latter application typically must deal with flows on the edge of flow breakdown (i.e. structural dynamic instabilities in the presence of dynamic flows and flows transitioning between states) where safety-of-flight is of paramount concern. Attention by the user-communities to the appropriately different standards to be applied to the validation or calibration of CFD methods in these two areas is required.

In conclusion, it is anticipated that unsteady CFD methods will emerge to be routinely used for aeroelastically challenging problems.

\section{References}

1. Agrawal, S.; Barnett, R. M.; and Robinson, B. A.: Investigation of Vortex Breakdown on a Delta Wing Using Euler and Navier-Stokes Equations, 67th AGARD Fluid Dynamics Panel Symposium on Vortex Flow Aerodynamic, Scheveningen, Netherlands, October 1-4, 1990.

2. Anderson, D. A.; Tannehill, J. C.; and Pletcher, R. H.: Computational Fluid Mechanics and Heat Transfer, McGrawHill Book Company, 1984.

3. Anderson, W. K.; Thomas, J. L.; and Rumsey, C. L.: Extension and Applications of Flux-Vector Splitting to Unsteady Calculations on Dynamic Meshes, AIAA Paper No. 87-1152, June, 1987.

4. Anderson, W. K.; and Batina, J. T.: Accurate Solutions, Parameter Studies, and Comparison for the Euler and Potential Flow Equations, Paper No. 15, AGARD 62nd Meeting of the Fluid Dynamics Panel Symposium on Validation of Computational Fluid Dynamics, AGARD CP 437, Vol. 1, Lisbon, Portugal, May 2-5, 1989.

5. Angelini, J. J.; Girodroux-Lavigne, P.; Grisval, J. P.; Le Balleur, J. C.; Mulak, P.; and Sides, J.: Unsteady Transonic Flow Computations for AGARD Two Dimensional and Three Dimensional Aeroelastic Configurations, ESA-TT-1020, December, 1986.

6. Angelini, J. J.; and Soize, C.: New Approach to Small Transonic Perturbations Finite Element Numerical Solving Method, Parts I and II. La Recherche Aerospatiale (English edition), No. 1989-2, 1989, pp. 1-41.

7. Anon: Military Specification; Airplane Strength and Rigidity; Flutter, Divergence, and Other Aeroelastic Instabilities. MIL-A-008870A (USAF), March 1971.

8. Anon: Unsteady Aerodynamics. AGARD-CP-227, 1978.

9. Anon: Boundary Layer Effects on Unsteady Airloads. AGARD-CP-296, 1981.

10. Anon: Unsteady Aerodynamics-Fundamentals and Applications to Aircraft Dynamics. AGARD-CP-386, 1985.

11. Anon: Transonic Unsteady Aerodynamics and its Aeroelastic Applications. AGARD-CP-374, 1985.

12. Anon: Aircraft Dynamic Loads Due to Flow Separation. AGARD-CP 483, September 1990.

13. Anon: Rapport de Synthese: Aeroelasticite Instationnaire Transsonique 6 EME Phase Configuration Aile du Falcon 900, Avions Marcel Dassault-Brequet Aviation, Document PBM.MI/YC-DTM-6233/88, Merignac, 7 October 1988.

14. Azevedo, J. L. F: Transonic Aeroelastic Analysis of Launch Vehicle Configurations. Ph.D. Dissertation, Department of Aeronautics and Astronautics, Stanford University, February 1988 (Also NASA CR 4186, October 1988).

15. Baldwin, B.; and Lomax, H.: Thin Layer Approximation and Algebraic Model for Separated Turbulent Flows, AIAA 
Paper No. 78-257, 1978.

16. Ballhaus, W. F.: Some Recent Progress in Transonic Flow Computations. VKI Lecture Series: Computational Fluid Dynamics, von Karman Institute for Fluid Dynamics, Rhode-St-Genese, Belgium, March 1976.

17. Ballhaus, W. F.; and Goorjian, P. M.: Implicit FiniteDifference Computations of Unsteady Transonic Flows about Airfoils. AIAA Journal, Vol. 15, No. 12, December 1977, pp. $1728-1735$.

18. Batina, J. T.: Unsteady Transonic Flow Calculations for Interfering Lifting Surface Configurations. Journal of Aircraft, Vol. 23, No. 5, May 1986, pp. 422-430. (Also NASA TM-86432, May 1985.)

19. Batina, J. T.: Unsteady Transonic Flow Calculations for Wing-Fuselage Configurations. Joumal of Aircraft, Vol 23, No. 12, December 1986, pp. 897-903.

20. Batina, J. T.: An Efficient Algorithm for Solution of the Unsteady Transonic Small-Disturbance Equation. Joumal of Aircraft, Vol 25, No. 7, July 1988, pp. 598-605. (Also NASA TM 89014, December 1986.)

21. Batina, J. T.; Seidel, D. A.; Bland, S. R.; and Bennett, R. M.: Unsteady Transonic Flow Calculations for Realistic Aircraft Configurations. Journal of Aircraft, Vol. 26, No. 2, February 1989, pp. 131-139. (Also NASA TM-89120, March 1987.)

22. Batina, J. T.: Unsteady Euler Airfoil Solutions Using Unstructured Dynamic Meshes. AIAA Journal, Vol. 28, No. 8, August 1990, pp. 1381-1388.

23. Batina, J. T.: Unsteady Euler Algorithm with Unstructured Dynamic Mesh for Complex-Aircraft Aeroelastic Analysis. AIAA Journal, Vol. 29, No. 3, March 1991, pp. 327-333.

24. Batina, J. T.: Unsteady Euler Airfoil Solutions Using Unstructured Dynamic Meshes. AlAA Journal, Vol. 28, No. 8. August 1990, pp. 1381-1388.

25. Bendiksen, O. O.; and Kousen, K. A.: Transonic Flutter Analysis Using the Euler Equations, AIAA Paper No. 87-0911, April, 1987.

26. Bennett, R. M.; and Batina, J. T.: Application of the CAP-TSD Unsteady Transonic Small Disturbance Program to Wing Flutter. Paper No. 89-003 in Proceedings of European Forum on Aeroelasticity and Structural Dynamics, DGLR Bericht 89-01, Aachen, FRG, April 17-19, 1989, pp. 25-34.

27. Bennett, R. M.; Eckstrom, C. V.; Rivera, J. A., Jr.; Dansberry, B. E.; Farmer, M. G.; and Durham, M. H.: Benchmark Aeroelastic Models Program - Description and Highlights from Initial Results. Paper No. 25, AGARD Specialists Meeting on Transonic Unsteady Aerodynamics and Aeroelasticity, San Diego, CA, October 9-11, 1991.

28. Berry, H. M.; Batina, J. T.; and Yang, T. Y.: Viscous Effects on Transonic Airfoil Stability and Response. Journal of Aircraft, Vol. 23, No. 5, May 1986, pp. 361-369.
29. Bharadvaj, B. K.: Computation of Steady and Unsteady Control Surface Loads in Transonic Flow. AIAA Paper No. 90-0935, April 1990.

30. Bland, S. R.: AGARD Two-Dimensional Aeroelastic Configurations. AGARD-AR-156, 1979.

31. Bland, S. R.: AGARD Three-Dimensional Aeroelastic Configurations. AGARD-AR-167, 1982.

32. Bland, S. R.; and Seidel, D. A.: Calculation of Unsteady Aerodynamics for Four AGARD Standard Aeroelastic Configurations, NASA TM 85817, May 1984.

33. Bland, S. R.; and Edwards, J. W.: Airfoil Shape and Thickness Effects on Transonic Airloads and Flutter. Journal of Aircraft, Vol. 21, No. 3, March 1984, pp. 209-217.

34. Bland, S. R.: Personal Computer Study of Finite-Difference Methods for the Transonic Small Disturbance Equation. Proceedings of the Third International Congress of Fluid Mechanics, Cairo, Egypt, January 2-4, 1990, Vol. II, pp. 527-543. (also NASA TM 102582, December 1989).

35. Bland, S. R.: Suggestions for CAP-TSD Mesh and TimeStep Input Parameters, NASA TM 104083, June 1991.

36. Bobbitt, P. J.: The Pros and Cons of Code Validation. AIAA Paper No. 88-2535. June 1988.

37. Borland, C. J.; and Nagaraja, K. S.: Applications and Improvements of XTRAN3S. WRDC-TR-89-3051, November 1989.

38. Carter, J. E.: A New-Boundary-Layer Interaction Technique for Separated Flows. AIAA Paper No. 79-1450, July 1979.

39. Chaderjian, N. M.; and Guruswamy, G. P.: Unsteady Transonic Navier-Stokes Computations for an Oscillating Wing Using Single and Multiple Zones, AIAA Paper No. 90-0313, January 1990.

40. Chen, P. C.; and Liu, D. D.: Unsteady Supersonic Computations of Arbitrary Wing-Body Configurations Including External Stores. Journal of Aircraft, Vol. 27, No. 2, February 1990 , pp. 108-116.

41. Chen, P. C.; Liu, D. D.; James, D. K.; and Potozky, A. S.: Further Studies of Harmonic Gradient Method for Supersonic Aeroelastic Applications. Paper No. 89-068 in Proceedings of European Forum on Aeroelasticity and Structural Dynamics, DGLR-Bericht 89-01, Aachen, FRG, April 1989, pp. 605-620.

42. Chyu, W. J.; Davis, S. S.; and Chang, K. S.: Calculation of Unsteady Transonic Flow Over an Airfoil. AIAA Journal, Vol. 19, No. 6, June 1981, pp. 684-690.

43. Chyu, W. J.; and Davis, S. S.: Numerical Studies of Unsteady Transonic Flow Over an Oscillating Airfoil. Paper No. 3 in AGARD CP-374, Transonic Unsteady Aerodynamics and its Aeroelastic Application, January 1985.

44. Cunningham, H. J.; Batina, J. T.; and Bennett, R. M.: Modern Wing Flutter Analysis by Computational Fluid Dynamics Methods. ASME Paper No. 87-WA/Aero-9, ASME 
Winter Annual Meeting, Boston, Massachusetts, December 13-18, 1987.

45. Damodaran, M.: Finite Volume Computation on Unsteady Inviscid Rotational Transonic Flows Past Airfoils in Rigid Body Motion, AIAA Paper 88-0006, January, 1988.

46. Deiwert, G. S.: Finite-Difference Simulation of Unsteady Interactive Flows. In Computational Methods in Viscous Flow, ed. by Habashi, W. G.; Pineridge Press, 1984, pp. 281-308.

47. Dobbs, S. K.; Miller, G. D.; and Stevenson, J. R.: SelfInduced Oscillation Wind Tunnel Test of a Variable Sweep Wing. AIAA Paper No. 85-0739, April 1985.

48. Dowell, E. H.: Nonlinear Aeroelasticity. AIAA Paper No. 90-1031, April 1990.

49. East, L. F.; Smith, P. D.; and Merryman, P. J.: Prediction of the Development of Separated Turbulent Boundary-Layers by the Lag-Entrainment Method. RAE-TR-77046, March 1977.

50. Edwards, D. E.; and Carter, J. E.: A Quasi-Simultaneous Finite Difference Approach for Strongly Interacting Flow, Third Symposium on Numerical and Physical Aspects of Aerodynamic Flows, Long Beach, California, January 21-24, 1985.

51. Edwards, J. W.; Bennett, R. M.; Whitow, W., Jr.; and Seidel, D. A.: Time-Marching Transonic Flutter Solutions Including Angle-of-Attack Effects. Joumal of Aircraft, Vol. 20, No. 11, November 1983, pp. 899-906.

52. Edwards, J. W; and Thomas, J. L.: Computational Methods for Unsteady Transonic Flow. Chapter 5 in Unsteady Transonic Aerodynamics, Ed. by D. Nixon, Vol. 120 of AIAA Progress in Astronautical and Aerodynamics, 1989, pp. 211-261. (Also AIAA Paper No. 87-0107).

53. Edwards, J. W.: Computational Aeroelasticity Challenges and Resources. In Transonic Unsteady Aerodynamics and Aeroelasticity, NASA CP 3022, 1989, Part 2, pp. 631-637.

54. Edwards, J. W.: Unsteady Aerodynamics: Physical Issues and Numerical Predictions. In Proceedings of the Third International Congress of Fluid Mechanics, Cairo, Egypt, January 2-4, 1990, Vol. I, pp. 99-115.

55. Edwards, J. W.: Assessment of Computational Prediction of Tail Buffeting. NASA TM 101613, January 1990.

56. Edwards, J. W.: Unsteady Airloads Due to Separated Flow on Airfoils and Wings. Paper No. 16 in AGARD-CP-483, Aircraft Dynamic Loads Due to Flow Separation, Sorrento, Italy, April 1990.

57. Farmer, M. G.; Hanson, P. W.; and Wynne, E. C.: Comparison of Supercritical and Conventional Wing Flutter Characteristics. NASA TM X-72837, May 1976.

58. Fenno, C. C., Jr.; Newman, P. A.; and Hassan, H. A.: Unsteady Viscous-Inviscid Interaction Procedures for Transonic Airfoils Using Cartesian Grids. Journal of Aircraft, Vol. 26, No. 8., August 1989, pp. 723-730.
59. Flores, J.; and Chaderjian, N. M.: The Numerical Simulation of Transonic Separated Flow About the Complete F-16A. AIAA Paper No. 88-2506, June 1988.

60. Fujii, K.; and Schiff, L. B.: Numerical Simulation of Vortical Flows Over a Strake-Deita Wing. AIAA Paper No. 87-1229, June 1987.

61. Fujii, K.; and Obayashi, S.: Navier-Stokes Simulations of Transonic Flows over a Wing-Fuselage Combination. AIAA Journal, Vol. 25, No. 12, December 1987, pp. $1587-1596$.

62. Gibbons, M. D.; Soistmann, D. L.; and Bennett, R. M.: Flutter Analysis of Highly Swept Delta Wings by Conventional Methods. NASA TM 101530, November 1988.

63. Girodroux-Lavigne, P.; and LeBalleur, J. C.: TimeConsistent Computation of Transonic Buffet Over Airfoils. 16th International Congress of the Aeronautical Sciences, Jerusalem, Israel, August 28-September 2, 1988.

64. Green, J. E.; Weeks, D. J.; and Brooman, J. W. F.: Prediction of Turbulent Boundary-Layers and Wakes in Compressible Flow by a Lag-Entrainment Method. RAE-TR-722311, January 1973.

65. Guruswamy, G. P.; and Goorjian, P. M.: Effects of Viscosity on Transonic-Aerodynamic and Aeroelastic Characteristics of Oscillating Airfoils. Joumal of Aircraft, Vol. 21, No. 9, September 1984, pp. 700-707.

66. Goorjian, P. M.; and Guruswamy, G. P.: Unsteady Transonic Aerodynamic and Aeroelastic Calculations About Airfoils and Wings. Paper No. 5 in Transonic Unsteady Aerodynamics and its Aeroelastic Applications, AGARD CP374, January 1985.

67. Guruswamy, G. P.: Time-Accurate Unsteady Aerodynamic and Aeroelastic Calculations of Wings Using Euler Equations. AIAA Paper No. 88-2281, April 1988.

68. Guruswamy, G. P.: Interaction of Fluids and Structures for Aircraft Applications, Computers and Structures, Vol. 30, No. 1/2, 1988, pp. 1-13.

69. Guruswamy, G. P.; and Goorjian, P. M.: Unsteady Transonic Aerodynamics and Aeroelastic Calculations at LowSupersonic Freestreams. Joumal of Aircraft, Vol. 25, No. 10, October 1988, pp. 955-961.

70. Guruswamy, G. P.; and Goorjian, P. M.: Unsteady Transonic Flow Simulation on a Full-Span-Wing Body Configuration. AIAA Joumal, Vol. 26, No. 12, December 1988, pp. $1450-1456$.

71. Henke, H.; Muller, U. R.; and Schultze, B.: A Viscous Inviscid Interaction Method for Use in Transonic Flutter Analysis, International Forum on Aeroelasticity and Structural Dynamics 1991, Aachen, Germany, June 3-6, 1991.

72. Hirose, N.; and Miwa, H.: Computational and Experimental Research on Buffet Phenomena of Transonic Airfoils, NAL TR 996T, 1988. 
73. Hitzel, S. M.: Wing Vortex-Flows Up Into VortexBreakdown. AIAA Paper No. 88-2518, June 1988.

74. Hounjet, M. H. L.: A Hybrid Field Panel/Finite Difference Method for 3-D Potential Unsteady Transonic Flow Calculations. AIAA Paper No. 83-1690, July, 1983.

75. Hounjet, M. H. L.; and Meijer, J. J.: Application of TimeLinearized Methods to Oscillating Wings in Transonic Flow and Flutter, Paper No. 11 in AGARD CP-374, Transonic Unsteady Aerodynamics and its Aeroelastic Applications, Toulouse, France, September, 1984.

76. Hounjet, M. H. L.: Calculation of Unsteady Subsonic and Supersonic Flow About Oscillating Wings and Bodies by New Panel Methods, Paper No. 89-009 in European Forum on Aeroelasticity and Structural Dynamics, DGLR-Bericht 89-01, Aachen, FRG, April 1989, pp. 85-104.

77. Houwink, R.: Unsteady Transonic Flow Computations, National Aerospace Laboratory NLR, Memorandum AE83-004 U, 1983.

78. Houwink, R.: Unsteady Viscous Transonic Flow Computations Using the LTRAN2-NLR Code Coupled with Green's Lag-Entrainment Method. Numerical and Physical Aspects of Aerodynamic Flows II, Chapter 15, ed. by T. Cebeci, Springer, New York, 1984.

79. Houwink, R.; and Veldman, A. E. P.: Steady and Unsteady Separated Flow Computations for Transonic Airfoils. AIAA Paper No. 84-1618, June 1984.

80. Howlett, J. T.: Efficient Self-Consistent Viscous-Inviscid Solutions for Unsteady Transonic Flow, Journal of Aircraft, Vol. 24, No. 11, November 1987, pp. 737-744.

81. Howlett, J. T.; and Bland, S. R.: Calculation of Viscous Effects on Transonic Flow For Oscillating Airfoils and Comparisons with Experiment. NASA TP-2731, September 1987.

82. Hsu, C.-H.; and Liu, C. H.: Upwind Navier-Stokes Solutions for Leading-Edge Vortex Flows, AIAA Paper No. 89-0265. January 1989.

83. Huband, G. W.; Rizzetta, D. P.; and Shang, J. J. S.: The Numerical Simulation of the Navier-Stokes Equations for an F-16 Configuration. AIAA Paper No. 88-2507, June 1988.

84. Ide, H.; and Shankar, V. J.: Unsteady Full Potential Aerœelastic Computations for Flexible Configurations. AIAA Paper No. 87-1238, June 1987.

85. Isogai, K.: Numerical Study of Transonic Flutter of a TwoDimensional Airfoil. NAL Report TR-716T, July 1980.

86. Isogai, K.: Calculation of Unsteady Transonic Potential Flow Over Oscillating Three-Dimensional Wings, NAL TR706T, March 1982.

87. Isogai, K.: The Development of Unsteady Transonic 3-D Full Potential Code and Its Aeroelastic Applications. Paper No. 17 in Transonic Unsteady Aerodynamics and Its Aeroelastic Applications, AGARD CP-374, January 1985.
88. Isogai, K.; and Suetsugu, K.: Numerical Simulation of Transonic Flutter of a Supercritical Wing, NAL Report TR726T, August 1982.

89. Johnson, E. H.; and Reymond, M. A.: Multidisciplinary Aeroelastic Analysis and Design Using MSC/NASTRAN, AIAA Paper No. 91-1097, April 1991.

90. Kandil, O. A.; and Chuang, J. A.: Unsteady Navier-Stokes Computations Past Oscillating Delta Wing at High Incidence, AIAA Paper No. 89-0081, January 1989.

91. Kandil, O. A.; and Salman, A. A.: Unsteady VortexDominated Flow Around Wings with Oscillating LeadingEdge Flaps, AIAA Paper No. 91-0435, January 1991.

92. Kandil, O. A.; and Salman, A. A.: Effects of Leading Edge Flap Frequency on Unsteady Flow Around Delta Wings, AIAA Paper No. 91-1796, June 1991.

93. Kleb, W.; Batina, J. T.; and Williams, M.: TemporalAdaptive Euler/Navier-Stokes Algorithm Unsteady Aerodynamic Analysis of Airfoils Using Unstructured Dynamic Meshes. AIAA Paper No. 90-1650, June 1990.

94. Kousen, K. A.; and Bendiksen, O. O.: Nonlinear Aspects of the Transonic Aeroelasticity Stability Problem, AIAA Paper No. 88-2306, April 1988.

95. Kousen, K. A.; and Bendiksen, O. O.: Limit Cycle Phenomena in Computational Transonic Aeroelasticity, AIAA Paper No. 89-1185, April 1989.

96. Krist, S. L.; Thomas, J. L.; Sellers, W. L., III; and Kjelgaard, S. O.: An Embedded Grid Formulation Applied to a Delta Wing. AIAA Paper No. 90-4029, January 1990.

97. Kwon, O. J.; and Sankar, L. N.: Viscous Flow Simulation of Fighter Aircraft, AIAA Paper No. 91-0278, January 1991.

98. LeBalleur, J. C.; and Girodroux-Lavigne, P.: A SemiImplicit and Unsteady Numerical Method of ViscousInviscid Interaction for Transonic Separated Flow. La Recherche Aerospatiale, 1984-1, English and French editions (1984), pp. 15-37.

99. LeBalleur, J. C.; and Girodroux-Lavigne, P.: A ViscousInviscid Interaction Method for Computing Unsteady Transonic Separation. Proc. 3rd Symposium on Numerical and Physical Aspects of Aerodynamic Flows, ed. by T. Cebici, Springer-Verlag, 1986, pp. 5-49. (Also ONERA TP 1985-5, 1985).

100. Lee, E. M.; and Batina, J. T.: Conical Euler Simulation and Active Suppression of Delta Wing Rocking Motion, Presented at the High-Angle-of-Attack Technology Conference, NASA Langley Research Center, Hampton, Virginia, October 30-November 1, 1990, Also NASA TM 102683, October 1990.

101. Lee, E. M.; and Batina, J. T.: Unsteady Euler Methodology for Unsteady Vortical Flows About Rolling Delta Wings, AIAA Paper No. 91-0730, January 1991. 
102. Liu, D. D.; Kao, Y. F.; and Fung, K.-Y.: Transonic Equivalent Strip Method for Aeroelastic Applications, Proceedings of the 2nd International Symposium on Aeroelasticity and Structural Dynamics, 1985, pp. 17-32.

103. Liu, D. D.; Kao, Y. F.; and Fung, K.-Y.: An Efficient Method for Computing Unsteady Transonic Aerodynamics of Swept Wings with Control Surfaces, AIAA Paper No. 85-4058, October, 1985.

104. Liu, D. D.; Lee, H. W.; and Chen, P. C.: Unsteady Subsonic Aerodynamics for Bodies and Wings with External Stores Including Wake Effect, Paper 91-060, International Forum on Aeroelasticity and Structural Dynamics, Aachen, FRG, June 3-5, 1991.

105. Mabey, D. G.; and Chambers, J. R.: Unsteady Aerodynamics - Fundamentals and Applications to Aircraft Dynamics, AGARD-AR-222, 1986.

106. Mabey, D. G.: A Review of Some Recent Research on Time-Dependent Aerodynamics. Aeronautical Journal, February, 1984, pp. 23-37.

107. Mabey, D. G.: Physical Phenomena Associated with Unsteady Transonic Flows, Chapter 1 in Unsteady Transonic Aerodynamics, ed. by D. Nixon, Vol. 120 of AIAA Progress in Astronautical and Aeronautics, 1989. pp. 1-55.

108. Magnus, R.; and Yoshihara, H.: The Transonic Oscillating Flap, AIAA Paper No. 76-327, July 1976.

109. Malone, J. B.; and Sankar, L. N.: Numerical Simulation of Two-Dimensional Unsteady Transonic Flows Using the Full Potential Equation. AIAA Journal, Vol. 22, No. 8, August 1984, pp. 1035-1041.

110. Meijer, J. J.; and Zwann, R. J.: Investigation of a SemiEmpirical Method to Predict Limit Cycle Oscillations of Modern Fighter Aircraft, AGARD CP-483, April, 1990.

111. Mortchelewitez, G. D.; and Sans, A. S.: Solution of 3D Euler Equations with Unstructured Meshes for Aeroelasticity Problems, Paper 91-066, Intemational Forum on Aeroelasticity and Structural Dynamics, Aachen, FRG, June 3-5, 1991

112. Mulak, P.; Meurzec, J. L.; and Angelini, J. J.: Extension and Use of Numerical Procedure for the Three Dimensional Unsteady Transonic Flows, Proceedings of the 2nd International Symposium on Aeroelasticity and Structural Dynamics, 1985, pp. 62-70.

113. Myers, M. R.; Guruswamy, P.; and Goorjian, P. M.: Flutter Analysis of a Transport Wing Using XTRAN3S, AIAA Paper No. 83-0922, 1983.

114. Mykytow, W. J.: Transonic Unsteady Aerodynamics and its Aeroelastic Applications, AGARD-CP-374, Addendum 1, June 1985.

115. Nakamichi, J.: Calculations of Unsteady Navier-Stokes Equations Around an Oscillatory 3-D Wing Using Moving Grid System, AIAA Paper No. 87-1158, June 1987.
116. Nayfeh, A. H.; Elzebda, J. M.; and Mook, D. T.: Analytical Study of the Subsonic Wing-Rock Phenomenon for Slender Delta Wings. Journal of Aircraft, Vol. 26, No. 9, September 1989, pp. 805-809.

117. Newsome, R. W.; and Kandil, O. A.: Vortical Flow Aerodynamics - Physical Aspects and Numerical Simulation, AIAA Paper No. 87-0205, January 1987.

118. Obayashi, S.; and Gunuswamy, G. P.: Unsteady ShockVorex Interaction on a Flexible Delta Wing, AIAA Paper No. 91-1109, April 1991.

119. Ominsky, D.; and Ide, H.: An Effective Flutter Control Method Using Fast, Accurate CFD codes, AIAA Paper No. 89-3468, August 1989.

120. Pearcy, H. H.; Haines, A. B.; and Osborne, J.: The Interaction Between Local Effects at the Shock and Real Separation, Paper No. 11 in Transonic Aerodynamics, AGARD-CP No. 356, 1968.

121. Petiau, C.; and Brun, S.: Tendances Actuelles De L'Analyse Aeroelastique Des Avions Militaires, Paper No. 2 in Static Aeroelastic Effects on High Performance Aircraft, AGARD-CP-403, 1986.

122. Pirzadeh, S.; and Whitfield, D. L.: Three-Dimensional Unsteady Transonic Viscous-Inviscid Interaction Using the Euler and Boundary-Layer Equation, AIAA Paper No. 88-2578, June 1988.

123. Pitt, D. M.; Fuglsang, D. F.; and Drouin, D. V.: Applications of XTRAN3S and CAP-TSD to Fighter Aircraft, AIAA Paper No. 90-1035, April 1990.

124. Rausch, R. D.; Batina, J. T.; and Yang. H. T. Y.: Spatial Adaption Procedures on Unstructured Meshes for Accurate Unsteady Aerodynamic Flow Computation, AIAA Paper No. 91-1106, April 1991.

125. Reisenthel, P. H.; and Nixon, D.: Prediction of Unsteady Separated Transonic Flow Around Missile Configurations, AIAA Paper No. 91-0601, January 1991.

126. Rivera, J. A., Jr.; Dansberry, B. E.; Farmer, M. G.; Eckstrom, C. V.; Seidel, D. A.; and Bennett, R. M.: Experimental Flutter Boundaries with Unsteady Pressure Distributions for the NACA 0012 Benchmark Model, AIAA Paper No. 91-1010, April 1991.

127. Rizk, Y. M.; Schiff, L. B.; and Gee, K.: Numerical Simulation of the Viscous Flow Around a Simplified F/A18 at High Angles of Attack, AIAA Paper No. 90-2999, August 1990.

128. Rizk, Y. M.; and Gee, K.: Numerical Prediction of the Unsteady Flowfield Around the F-18 Aircraft at Large Incidence, AIAA Paper No. 91-0020, January 1991.

129. Rizzetta, D. P.: Procedures for the Computation of Unsteady Transonic Flows Including Viscous Effects, NASA CR 166249, January 1982. 
130. Robinson, B. A.; Batina, J. T.; and Yang, H. T. Y.: Aeroelastic Analysis of Wings Using the Euler Equations with a Deforming Mesh, AIAA Paper No. 90-1032, April 1990.

131. Rodden, W. P.; Giesing, J. P.; and Kalman, T. P.: New Developments and Applications of the Subsonic Doublet-Lattice Method for Nonplanar Configurations, $\mathrm{Pa}$ per No. 4 in the Symposium on Unsteady Aerodynamics for Aeroelastic Analyses of Interfering Surfaces, AGARD CP-80-71, Part 2, 1971.

132. Rodman, L. C.; Nixon, D.; and Huttsell, L. J.: Modifications to Transonic Flow Codes for Unsteady Perturbations Around an Experimental Mean. Journal of Aircraft, Vol. 26, No. 8, August 1989, pp. 765-771.

133. Rowe, W. S.; and Cunningham, H. J.: On the Convergence of Unsteady Generalized Aerodynamic Forces. Joumal of Aircraft, Vol. 21, No. 6, June 1984, pp. 420-427.

134. Rumsey, C. L.; and Anderson, W. K.: Some Numerical and Physical Aspects of Unsteady Navier-Stokes Computations Over Airfoils Using Dynamic Meshes, AIAA Paper No. 88-0329, January 1988.

135. Sankar, L. M.; Malone, J. B.; and Tassa, Y.: An Implicit Conservative Algorithm for Steady and Unsteady Transonic Potential Flows, AIAA Paper No. 81-1016, June 1981.

136. Sankar, L. M.; and Malone, J. B.: A Numerical Solution Procedure for Steady and Unsteady Transonic Potential Flows. Advances in Computational Transonics, Recent Advancements in Numerical Methods in Fluids, Vol. IV, W. G. Habashi, Editor, Pineridge Press, 1985.

137. Sankar, L. M.; Ruo, S. Y.; and Malone, J. B.: Application of Surface Transpiration in Computational Aerodynamics, AIAA Paper No. 86-0511, January 1986.

138. Schippers, J.: Numerical Integration of the Unsteady FullPotential Equation with Applications of Transonic Flow about a 2D Airfoil, NLR MP 84022 U, 1984.

139. Schuster, D. M.; Vadyak, J.; and Atta, E.: Static Aeroelastic Analysis of Fighter Aircraft Using a Three-Dimensional Navier-Stokes Algorithm. Journal of Aircraft, Vol. 27, No. 9. September 1990, pp. 820-825.

140. Seidel, D. A.; Bennett, R. M.; and Whitlow, W., Jr.: An Exploratory Study of Finite Difference Grids for Transonic Unsteady Aerodynamics, AIAA Paper No. 83-0503, January 1983.

141. Seidel, D. A.; Sandford, M. C.; and Eckstrom, C. V.: Measured Unsteady Transonic Aerodynamic Characteristics of an Elastic Supercritical Wing. Journal of Aircraft, Vol. 24, No. 4, April 1987, pp. 225-230.

142. Shankar, V.; Ide, H.; Gorski, J.; and Osher, S.: A Fast Time-Accurate Unsteady Full-Potential Scheme, AIAA Paper No. 85-1512, July 1985.

143. Shieh, T. H.; Schoen, J. G.; and Fung, K.-Y.: Techniques for Accurate, Efficient Computation of Unsteady Transonic Flow, AIAA Paper No. 91-0597, January 1991.
144. Silva, W. A.; and Bennett, R. M.: Using Transonic Small Disturbance Theory for Predicting the Aeroelastic Stability of a Flexible Wind-Tunnel Model, AIAA Paper No. 90-1033, April 1990. (Also NASA TM 102617, March 1990.).

145. Sotomayer, W. A.; and Borland, C. J.: Numerical Computation of Unsteady Transonic Flow About Wings with Flaps, AIAA Paper No. 85-1712, July 1985.

146. Sotomayer, W. A.; and Sankar, L. N.; and Malone, J. B.: A Comparison of Numerical Algorithms for Unsteady Transonic Flow. Computer Methods in Applied Mechanics and Engineering, Vol. 64, 1987, pp. 237-265.

147. Steger, J. L.: Implicit Finite-Difference Simulation of Flow about Arbitrary Two-Dimensional Geometries. AIAA Joumal, Vol. 16, No. 7, July 1978, pp. 679-686.

148. Steger, J. L.; and Bailey, H. E.: Calculation of Transonic Aileron Buzz. AIAA Journal, Vol. 18, No. 3, March 1980, pp. 249-255.

149. Steiginga, A.; and Houwink, R.: Correlation of Experimental and Quasi-3D Theoretical Airloads on the Oscillating LANN Supercritical Wing Model, AFWAL-TR83-3050, December 1982.

150. Thomas, J. L.; Taylor, S. L.; and Anderson, W. K.: Navier-Stokes Computations of Vortical Flows Over Low Aspect Ratio Wings, AIAA Paper No. 87-0207, January 1987.

151. Thomas, J. L.; Walters, R. W.; Reu, T.; Ghaffari, F; Weston, R. P.; and Luckring, J. M.: A Patched-Grid Algorithm for Complex Configurations Directed Towards the F/A-18 Aircraft, AIAA Paper No. 89-0121, January 1989.

152. Tijdeman, J.: Investigations of the Transonic Flow Around Oscillating Airfoils, National Aerospace Laboratory NLR, The Netherlands, NLR TR 77090U, 1978.

153. Transonic Unsteady Aerodynamics and Aeroelasticity. Proceedings from a conference at NASA Langley Research Center, May 20-22, 1987, NASA CP 3022, 1989, 2 Parts.

154. Triplett, W. E.: Pressure Measurements on Twin Vertical Tails in Buffeting Flow, AFWAL-TR-82-3015, Volume I, April 1982.

155. Unsteady Transonic Aerodynamics. ed. by Nixon, D.; Volume 120 in AIAA Progress in Astronautics and Aeronautics, 1989.

156. Vadyak, J.; and Schuster, D. M.: Navier-Stokes Simulation of Burst Vortex-Flowfields for Fighter Aircraft at High Incidence, AIAA Paper No. 89-2190, July 31-August 2, 1989.

157. Vatsa, V. N.; and Wedan, B. W.: Development of an Efficient Multigrid Code for a 3-D Navier-Stokes Equations, AIAA 89-1791, June 1989. 
158. Veldman, A. E. P.: New Quasi-Simultaneous Method to Calculate Interacting Boundary Layers. AIAA Journal, Vol. 19, No. 1, January 1981, pp. 79-85.

159. Vinh, L. S.; Edwards, J. E.; Seidel, D. A.; and Batina, J. T.: Transonic Stability and Control of Aircraft Using CFD Methods, AIAA Paper No. 88-4374, August 1988.

160. Voss, R.: Calculation of Unsteady Transonic Flow About Oscillating Wings by a Field Panel Method, Proceedings of the 3rd GAMMS Seminar, January, 1987, pp. 232-242.

161. Weatherill, W. H.; and Ehlers, F. E.: Development and Application of a Program to Calculate Transonic Flow Around an Oscillating Three-Dimensional Wing Using Finite Difference Procedures, NASA CR 181744, February 1989.

162. Whitfield, D. L.; Janus, J. M.; and Arabshahi, A.: Unsteady Euler Solutions on Dynamic Blocked Grids for Complex Configurations, AGARD CP-464, May 1989.

163. Whitlow, W., Jr.: Application of a Nonisentropic Full Potential Method to AGARD Standard Airfoils, AIAA Paper No. 88-0710, January 1988.

164. Wu, J. C.; Kaza, K. R. V.; and Sankar, L. N.: A Technique for the Prediction of Airfoil Flutter Characteristics in Separated Flow, AIAA Paper No. 87-0910, 1987.

165. Wong, Y. S.; and Lee, B. H. K.: Development of a. Three Dimensional Unsteady Transonic Aerodynamics Computer Code for Flutter Analysis, Paper No. ICAS-90-1.1.4, September 1990.

166. Yates, E. C., Jr.: AGARD Standard Aeroelastic Configurations for Dynamic Response I-Wing 445.6, AGARD-R765, July 1988.

167. Zwann, R. J.: Aeroelastic Problems of Wings in Transonic Flow, VKI Lecture Series on Unsteady Airloads and Aeroelastic Problems in Separated and Transonic Flow, Rhodes-Saint-Genese, Belgium, March 1981. (Also NLR MP $81005 U)$.

168. Zwann, R. J.: Verification of Calculation Methods for Unsteady Airloads in the Prediction of Transonic Flow, NLR MP 84014 U, May 1984.

169. Zimmerman, H.; and Vogel, S.: Applications of Transonic Unsteady Methods for Calculation of Flutter Airloads, Proceedings of the 2nd International Symposium on Aeroelasticity and Structural Dynamics, 1985, pp. 47-61.

170. Zimmerman, H.: Comparison Between 2D Transonic Flutter Calculations in the Time and Frequency Domain, AGARD Report No. 749, October 1986.

171. Zimmerman, N. H.; Ferman, M. A.; and Yurkovich, R. N.: Prediction of Tail Buffet Loads for Design Application, AIAA Paper No. 89-1378, April 1989. 

Public redorting ourden for this collection of information is estimated to average 1 hour per response, including the time tor reviewing instructions. searching existing cata sources. gathering and maintaining the data needed, and completing and reviewing the collection of intormation. Send comments regarding this burden estimate or any other aspect of this Davis Highway, Suite 1204, Arlington. VA 22202-4302, and to ine Office of Management and Budget. Paperwork Reducion Project (0704-0188), Washington, DC 20503.

\begin{tabular}{|l|c|c|}
\hline 1. AGENCY USE ONLY (Leave blank) & $\begin{array}{c}\text { 2. REPORT DATE } \\
\text { January } 1992\end{array}$ & $\begin{array}{c}\text { 3. REPORT TYPE AND DATES COVERED } \\
\text { Technical Memorandum }\end{array}$ \\
\hline
\end{tabular}

4. TITLE AND SUBTITLE

Current Status of Computational Methods for Transonic

Unsteady Aerodynamics and Aeroelastic Applications
5. FUNDING NUMBERS

$505-63-50-12$

6. AUTHOR(S)

John W. Edwards

John B. Malone

7. PERFORMING ORGANIZATION NAME(S) AND ADDRESS(ES)

NASA Langley Research Center

8. PERFORMING ORGANIZATION

Hampton, VA 23665-5225 REPORT NUMBER

9. SPONSORING / MONITORING AGENCY NAME(S) AND ADDRESS(ES)

National Aeronautics and Space Administration

Washington, DC 20546-0001

10. SPONSORING / MONITORING AGENCY REPORT NUMBER

NASA TM-104191

11. SUPPLEMENTARY NOTES

Presented at the AGARD Structures and Materials Panel Specialist's Meeting on Transonic Unsteady Aerodynamics and Aeroelasticity, in San Diego, California october 9-11. 1991. Paper No. 1 12a. DISTRIBUTION/AVAILABILITY STATEMENT

Unclassified-Unlimited

Subject Category 02

\section{ABSTRACT (Maximum 200 words)}

The current status of computational methods for unsteady aerodynamics and aeroelasticity is reviewed. The key features of challenging aeroelastic applications are discussed in terms of the flowfield state: low-angle high speed flows and high-angle vortex-dominated flows. The critical role played by viscous effects in determining aeroelastic stability for conditions of incipient flow separation is stressed. The need for a variety of flow modeling tools, from linear formulations to implementations of the Navier-Stokes equations, is emphasized. Estimates of computer run times for flutter calculations using several computational methods are given. Applications of these methods for unsteady aerodynamic and transonic flutter calculations for alrfoils, wings and configurations are summarized. Finally, recommendations are made concerning future research directions.

14. SUBJECT TERMS

Transonic Unsteady Aerodynamics Finite Difference, Aeroelasticity

\begin{tabular}{|l|l|}
\hline 17. SECURITY CLASSIFICATION & $\begin{array}{l}\text { 18. SECURITY CLASSIFICATION } \\
\text { OF REPORT }\end{array}$ \\
OF THIS PAGE \\
Unclassified
\end{tabular}$\quad$\begin{tabular}{l} 
Unclassified \\
\hline
\end{tabular}

19. OF ABSTRACT
Unclassified 
. 